(c) 1996 Kluwer Academic Publishers. Printed in the Netherlands.

\title{
Perturbation of Dirichlet Forms by Measures
}

\author{
PETER STOLLMANN ${ }^{1}$ and JÜRGEN VOIGT ${ }^{2}$ \\ ${ }^{1}$ Fachbereich Mathematik, Universität Frankfurt, D-60054 Frankfurt, Germany \\ ${ }^{2}$ Fachrichtung Mathematik, Technische Universität Dresden, D-0I062 Dresden, Germany
}

(Received: 26 August 1993; accepted: 1 August 1994)

\begin{abstract}
Perturbations of a Dirichlet form $h$ by measures $\mu$ are studied. The perturbed form $h-\mu_{-}+$ $\mu_{+}$is defined for $\mu_{-}$in a suitable Kato class and $\mu_{+}$absolutely continuous with respect to capacity. $L_{p}$-properties of the corresponding semigroups are derived by approximating $\mu_{-}$by functions. For treating $\mu_{+}$, a criterion for domination of positive semigroups is proved. If the unperturbed semigroup has $L_{p}-L_{q}$-smoothing properties the same is shown to hold for the perturbed semigroup. If the unperturbed semigroup is holomorphic on $L_{\mathrm{I}}$ the same is shown to be true for the perturbed semigroup, for a large class of measures.
\end{abstract}

Mathematics Subject Classifications (1991): Primary 47D07; Secondary 31C25.

Key words: Dirichlet form, measure perturbation, substochastic semigroup, capacity, smooth measures.

\section{Introduction}

If one wants to define a Schrödinger operator $-\frac{1}{2} \Delta+V$ in $L_{2}\left(\mathbb{R}^{n}\right)$ for a potential so singular that there is no direct way interpretation it is meanwhile traditional to use the theory of forms in order to define the operator. The term of the form associated with $V$ can be thought of as generated by the measure $V \lambda^{n}$ (where $\lambda^{n}$ is $n$-dimensional Lebesgue measure). It is the aim of this paper to investigate properties of the operator obtained if $V \lambda^{n}$ is replaced by a more general measure $\mu$.

The context in which the operator discussed above can be understood best is the evolution associated with the heat equation with absorption

$$
u_{t}=\frac{1}{2} \Delta u-V u
$$

where now the absorption-excitation rate $V$ should be replaced by the absorptionexcitation 'distribution' $\mu$. The effects of absorption-excitation can be assessed by looking at the Feynman-Kac formula

$$
e^{t\left(\frac{1}{2} \Delta-V\right)} f(x)=E_{x}\left\{\exp \left(-\int_{0}^{t} V(b(s)) \mathrm{d} s\right) f(b(t))\right\}
$$

describing the solution of the Cauchy problem for (1) with initial value $f$, where $b$ runs through the continuous paths, and $E_{x}$ is expection with respect to Wiener 
measure on paths starting at $x$. From (2) it is intuitively clear that $V$ cannot be replaced by measures concentrated on sets which are not 'seen' by the Wiener process. Also, it is apparent that, in order to generate a reasonable evolution, the restriction on the negative part of the measure must be more incisive than on the positive part.

More generally, we shall treat the problem discussed so far in the context where $-\frac{1}{2} \Delta$ is replaced by a selfadjoint operator $H$ associated with a regular Dirichlet form $\mathfrak{h}$. The positive Borel measures $\mu$ generating a closed form $\mathfrak{h}+\mu$ are those which are 'absolutely continuous' with respect to the capacity generated by $\mathfrak{h}$. (We point out at once that the notion of 'Borel measure' does not include the property that the measure of compact sets should be finite.) The positive measures $\mu$ such that $\mathfrak{h}-\mu$ generates a semigroup acting in all $L_{p}$-spaces are those in an extended Kato class.

The main topic of this paper is to study different aspects of the semigroup $\left(e^{-t H_{\mu}} ; t \geqslant 0\right)$, where $H_{\mu}$ is the operator associated with $\mathfrak{h}+\mu$. The fundamental principle in this investigation is to approximate $\mu$ by functions and transfer the properties known for the approximating objects. In the context of general Dirichlet forms, the operation of convolution is not available; in this case, smoothing of $\mu$ is achieved by applying resolvents of $H$ suitably.

Measure perturbations of Dirichlet forms have been studied in increasing generality and with different aims during the past years; cf. [BM], [ABR], [AM], [BEKS], [Stu]. One of the differences to all of these papers (except [BEKS]) is that our approach is operator theoretical in spirit (even if we use, of course, potential theory of Dirichlet forms), whereas in the mentioned papers probabilistic methods are used. As a side effect we can dispense with some assumptions which are needed in order to construct a process associated with a Dirichlet form; cf. Section 1.

The paper is organized as follows:

1. The Kato class of measures

2. Approximation of measures in $\hat{S}_{K} \cap S_{0}$

3. Approximation of measures in $\hat{S}_{K}$

4. Perturbation by general measures

5. $L_{p}-L_{q}$-smoothing

6. Holomorphy of $L_{1}$-semigroups

Appendix A. A form convergence theorem

Appendix B. On the comparison of symmetric semigroups

In Section 1 we introduce our setup concerning Dirichlet forms $\mathfrak{h}$. We define the perturbed form $\mathfrak{h}+\mu$ for $\mu \in M_{0}$, discuss smooth measures and introduce the extended Kato class $\hat{S}_{K}$ of measures.

In Sections 2 and 3 we study $\mathfrak{h}-\mu$ for measures $\mu \in \hat{S}_{K}$ with $c(\mu)<1$. The ultimate aim is to show that, with the associated selfadjoint oper- $H_{-\mu}$, the semigroup $\left(e^{-t H_{-\mu}} ; t \geqslant 0\right)$ acts also on all $L_{p}$-spaces, for $1 \leqslant p<\infty$ (Theorem 3.3). In Section 2 the approximation procedure is described. The first step does not yield 
approximation of general measures $\mu \in \hat{S}_{K}$ by functions, but only of those which additionally are of finite energy integral, $\mu \in S_{0}$. This, however, turns out to be sufficient since measures in $\hat{S}_{K}$ can be cut off, yielding measures in $S_{0}$.

In Section 4 we study the form $h-\mu_{-}+\mu_{+}$where $\mu_{-} \in \hat{S}_{K}, c\left(\mu_{-}\right)<1$, and $\mu_{+} \in M_{0}$. Again it is shown that the corresponding semigroup acts also in $L_{p}$, for $1 \leqslant p<\infty$.

In the remaining two sections we require additional properties of $h$. In Section 5 it is assumed that the unperturbed semigroup has the property that $e^{-t H}$ maps $L_{1}$ to $L_{\infty}$ for all $t>0$. It is shown that then the semigroups obtained in Section 4 enjoy the same property. In Section 6 we assume that the unperturbed semigroup is holomorphic in $L_{1}$. Then it is shown that under rather general conditions the perturbed semigroup has this property as well.

Finally, in Appendix A we prove an abstract form convergence result which is used in Section 2, and in Appendix B we establish a criterion for domination of positive semigroups which is needed in Section 4.

\section{The Kato Class of Measures}

Throughout the whole paper, we shall need the following assumptions and notations.

$X$ will denote a locally compact Hausdorff space, $m$ will be a Radon measure on $X$ with $\operatorname{supp} m=X$. $\mathcal{B}$ will denote the Borel $\sigma$-algebra on $X$. Further we assume that a regular Dirichlet form $b$ in $L_{2}(X, m)$ is given; its domain will be denoted by $D$, and the corresponding scalar product on $D$ is $(\cdot \mid \cdot)_{\mathfrak{g}}:=(\mathfrak{h}+1)[\cdot, \cdot]$. (For Dirichlet forms and their properties we refer to [Fu]; in particular we recall that 'regular' means that $D \cap C_{c}(X)$ is dense in $D$, i.e. $D \cap C_{c}(X)$ is a core for $\mathfrak{h}$, and additionally that $D \cap C_{c}(X)$ is dense in $C_{c}(X)$ with the supremum norm. Throughout the paper we fix the scalar field $\mathbb{K}$ which may be $\mathbb{R}$ or $\mathbb{C}$; accordingly the function spaces are real or complex.) By $H$ we denote the selfadjoint operator corresponding to $h$, and by $\left(U(t):=e^{-t H} ; t \geqslant 0\right)$ the corresponding semigroup. Recall in particular that, by the Beurling-Deny criteria, $U(t)$ acts as a positivity preserving contraction on all $L_{p}(m), 1 \leqslant p \leqslant \infty$, for all $t \geqslant 0$. By $D^{*}$ we denote the conjugate linear continuous functionals on $D$, dualized over the scalar product of $L_{2}(X, m)$, so that $D \subset L_{2}(X, m) \subset D^{*}$. Finally, $\operatorname{cap}(\cdot)$ will denote the capacity defined by $h$.

We note that our assumptions concerning $X$ are more general than those used in $[\mathrm{Fu}]$; in fact, it is not clear whether one can associate a suitable process with h under these conditions. The results we shall quote from $[\mathrm{Fu}]$ are valid in this generality, however, since their proofs obviously carry over.

Concerning Dirichlet forms we also mention [BH], [MR]. Dirichlet forms provide a framework for a unified treatment of a variety of operators $H$ of physical interest: Laplace operators on (open subsets of) $\mathbb{R}^{n}$ or manifolds ([Da1]), relativistic Hamiltonians of the form $H=\sqrt{-\Delta+m^{2}}-m$ ([CMS]), or certain pseudodiffer- 
ential operators $([\mathrm{HJ}],[\mathrm{J}])$. Further we mention the application to Feller generators ([Dem]).

We recall that any $u \in D$ admits quasi-continuous versions, denoted by $u^{\sim}$, two of which coincide quasi-every where.

We define

$$
\begin{aligned}
M_{0}:= & \{\mu: \mathcal{B} \rightarrow[0, \infty] ; \mu \sigma \text {-additive, and } \mu(N)=0 \\
& \text { for every set } N \in \mathcal{B} \text { of zero capacity }\}
\end{aligned}
$$

A $\sigma$-additive set function $\mu: \mathcal{B} \rightarrow[0, \infty]$ may also be called a Borel measure on $X$. A Radon measure $\mu$ on $X$ will be an inner regular Borel measure such that $\mu(K)<\infty$ for all compact $K$.

For $\mu \in M_{0}$, integrals with functions in $D$ can be defined since they do not depend on the choice of the quasi-continuous version. It was shown in [Sto2], that for each $\mu \in M_{0}$ a closed form in $\left(D,(\cdot \mid \cdot)_{h}\right)$ is defined by

$$
\begin{aligned}
& D(\mu):=\left\{u \in D ; u^{\sim} \in \mathcal{L}_{2}(X, \mu)\right\}, \\
& \mu[u, v]:=\int u^{\sim} \cdot \overline{v^{\sim}} \mathrm{d} \mu,
\end{aligned}
$$

and consequently a closed form $\mathfrak{b}+\mu$ in $L_{2}(X, m)$ is defined by

$$
\begin{aligned}
& D(\mathfrak{h}+\mu)=D(\mu), \\
& (\mathfrak{h}+\mu)[u, v]:=\mathfrak{h}[u, v]+\mu[u, v] .
\end{aligned}
$$

We note at this point that the corresponding definition in $[A M]$ is incorrect: Despite the fact that [AM; (3.1)] defines a sesquilinear form on $L_{2}(|\mu|+m)$ it cannot be considered as a form on $L_{2}(m)$, in general.

Our next result will show that the class $M_{0}$ is the natural set which can be used in order to generate closed forms. Let $R$ denote the (order complete) lattice of positive Radon measures on $X$. Then $R \cap M_{0}$ is a band in $R$, and therefore each $\mu \in R$ has a unique decomposition, called the Riesz decomposition, $\mu=\mu_{\tau}+\mu_{s}$ with $\mu_{T} \in R \cap M_{0}$, and $\mu_{s}$ disjoint to $R \cap M_{0}$, i.e., $\nu \in R \cap M_{0}$ and $\nu \leqslant \mu_{s}$ implies $\nu=0$. (For the notions concerning vector lattices which were used above we refer to [Sch; Chapter II]. These notions apply, in fact, to vector lattices; we have employed them for the corresponding positive cones.)

1.1. PROPOSITION. Let $\mu$ be a Radon measure on $X$, and let $\mu=\mu_{r}+\mu_{s}$ be the decomposition of $\mu$ just described.

(a) For each compact $K \subset X$ there exists a set $N \subset K, N \in \mathcal{B}$ such that $\operatorname{cap}(N)=0, \mu_{s}(K \backslash N)=0$. (Compare [FST; Lemma 2.1]; $\mu_{r}$ is called the smooth part of $\mu$.) 
(b) The regular part (cf. [Si1], [RS; supplement to VIII.7]) $\left(\mathfrak{h}+\mu \mid D \cap C_{c}(X)\right)_{\tau}$ of $\mathfrak{h}+\mu$ restricted to $D \cap C_{c}(X)$ is given by $\mathfrak{h}+\mu_{r}$.

Proof. (a) (see [FST; loc. cit.]). Let

$$
\mathcal{N}:=\{N \subset K ; N \in \mathcal{B}, \operatorname{cap}(N)=0\} .
$$

Then there exists $N \in \mathcal{N}$ such that

$$
\mu_{s}(N)=\sup _{N^{\prime} \in \mathcal{N}} \mu_{s}\left(N^{\prime}\right)
$$

(The proof is as in the proof for the Lebesgue decomposition; cf. [Ba; proof of Theorem 17.13, p. 120 f.J.) This implies $\mu_{s}\left(N^{\prime}\right)=0$ for all $N^{\prime} \in \mathcal{N}$ with $N^{\prime} \cap N=\emptyset$, i.e., $\chi_{K \backslash N} \mu_{s} \in M_{0}$. Since $\mu_{s}$ is disjoint to $M_{0} \cap R$ we conclude $\chi_{K \backslash N} \mu_{s}=0$.

(b) Clearly, $\left(\mathfrak{h}+\mu \mid D \cap C_{c}(X)\right)_{r}=: \mathfrak{t}$ dominates $\mathfrak{h}+\mu_{r}$. To prove $\mathfrak{t} \leqslant \mathfrak{h}+\mu_{r}$, let $\varphi \in D \cap C_{c}(X), K:=\operatorname{supp} \varphi$, and choose $N$ as in (a). The regularity of $\mu_{s}$ implies the existence of a sequence of compact sets $K_{1} \subset K_{2} \subset \cdots \subset N$ such that $\mu_{s}\left(N \backslash \bigcup_{n \in \mathbb{N}} K_{n}\right)=0$. This implies that there exists a sequence $\left(\psi_{n}\right)$ in $D \cap C_{c}(X)$ such that $0 \leqslant \psi_{n} \leqslant 1, \chi_{n}(x)=1$ for all $x \in K_{n},\left\|\psi_{n}\right\|_{h} \rightarrow 0$. Let $\varphi_{n}:=\varphi\left(1-\psi_{n}\right)$. Then $\varphi_{n} \rightarrow \varphi$ weakly in $\left(D,(\cdot \mid \cdot)_{h}\right)$; therefore there exists a sequence $\left(\hat{\varphi}_{n}\right), \hat{\varphi}_{n}$ a convex combination of $\left\{\varphi_{m} ; m \geqslant n\right\}(n \in \mathbb{N})$, converging to $\varphi$ in $D$ (weak and strong closure coincide for convex sets); without restriction we may assume $\varphi_{n} \rightarrow \varphi$ in $D$. By construction, $\varphi_{n} \rightarrow 0$, $\mu_{s}$-a.e., and so $\mu_{s}\left[\varphi_{n}\right] \rightarrow 0$ for $n \rightarrow \infty$. Now the closedness of $t$ implies

$$
\begin{aligned}
\mathbf{t}[\varphi] & \leqslant \lim \mathbf{t}\left[\varphi_{n}\right] \leqslant \lim (\mathfrak{h}+\mu)\left[\varphi_{n}\right] \\
& =\lim \left(\mathfrak{h}+\mu_{\tau}\right)\left[\varphi_{n}\right]=\left(\mathfrak{h}+\mu_{r}\right)[\varphi] .
\end{aligned}
$$

In case that $X$ is countable at infinity, a measure $\mu$ is called smooth, if there exists an increasing sequence of compact sets, $\left(F_{n}\right)$, such that $\mu\left(F_{n}\right)<\infty$ and $\operatorname{cap}\left(K \backslash F_{n}\right) \rightarrow 0$ for every compact $K^{r} \subset X$; cf. [Fu; p. 72]. For arbitrary locally compact spaces this condition is not suitable, since it allows only measures which 'live' on a $K_{\sigma}$-subset of $X$. We shall now give a notion of smoothness which appears to be more appropriate for our general context.

1.2. DEFINITION. Let $K \subset X$ be compact. Then a sequence $\left(F_{n}\right)$ of closed sets in $X$ (not necessarily $F_{n} \subset K$ ) is called a $K$-nest, if $\operatorname{cap}\left(K \backslash F_{n}\right) \rightarrow 0$ for $n \rightarrow \infty$.

1.3. DEFTNITION. A measure is called smooth, if $\mu \in M_{0}$ and if for every $K \subset X$ there exists a $K$-nest $\left(F_{n}\right)$ such that $\mu\left(F_{n}\right)<\infty$ for all $n \in \mathbb{N}$. The set of smooth measures will be denoted by $S$. 
To illustrate the usefulness of these notions and to verify that our definition coincides with Fukushima's in case that $X$ is countable at infinity, we state the following results.

1.4. THEOREM (cf. [Sto2; Theorem 2.1]). Let $\varphi \in D \cap C_{c}(X), K:=\operatorname{supp} \varphi,\left(F_{n}\right)$ a K-nest. Then

$$
\varphi \in{\left.\overline{\operatorname{lin}\left\{f \in D ; \exists n \in \mathbb{N}: 0 \leqslant f^{\sim} \leqslant \chi F_{n}\right.}\right\}}^{D} .
$$

1.5. THEOREM. For $\mu \in M_{0}$, the following conditions are equivalent:

(i) $\mu$ is smooth.

(ii) $D(\mu)$ is dense in $\left(D,(\cdot \mid \cdot)_{h}\right)$.

For the proof, see [Sto2]; for measures of the form $V m$ the proof was given in [Sto1].

1.6. THEOREM (cf. [Fu; Theorem 3.2.3, p. 73]). Let $\mu$ be a Borel measure. Then the following are equivalent:

(i) $\mu$ is smooth.

(ii) For every compact $K$ there exists a $K$-nest $\left(F_{n}\right)$, such that $\chi_{F_{n}} \mu \in S_{0}$ for all $n \in \mathbb{N}$.

For the proof, see [Fu; p. 73]. Here we have used the notation $S_{0}$ for the class of measures of finite energy integral, i.e.,

$$
S_{0}:=\left\{\mu \in M_{0} ; D \ni u \mapsto \int u^{\sim} \mathrm{d} \mu \text { is continuous with respect to }\|\cdot\|_{\natural}\right\} .
$$

In order to introduce the extended Kato class we use the following mapping for $\mu \in M_{0}, \alpha>0$ :

$$
\begin{aligned}
& \Phi(\mu, \alpha): C_{c}(X)_{+} \rightarrow[0, \infty] \\
& \Phi(\mu, \alpha) f:=\int_{X}\left((H+\alpha)^{-1} f\right)^{\sim} \mathrm{d} \mu\left(=\int_{X}\left(\int_{0}^{\infty} e^{-\alpha t} U(t) f \mathrm{~d} t\right)^{\sim} \mathrm{d} \mu\right) .
\end{aligned}
$$

We define the extended Kato class

$$
\begin{aligned}
\hat{S}_{K}:= & \left\{\mu \in M_{0} ; \exists \alpha>0: \Phi(\mu, \alpha)\right. \text { extends to a bounded linear } \\
& \text { functional on } \left.L_{1}(X, m)\right\},
\end{aligned}
$$

and for $\mu \in \hat{S}_{K}, \alpha>0$ we define

$$
c_{\alpha}(\mu):=\|\Phi(\mu, \alpha)\|_{\infty}\left(=\|\Phi(\mu, \alpha)\|_{L_{1}(X, m)^{\prime}}\right),
$$




$$
c(\mu):=\inf _{\alpha>0} c_{\alpha}(\mu)=\lim _{\alpha \rightarrow \infty} c_{\alpha}(\mu)
$$

The resolvent equation implies $c_{\alpha}(\mu)<\infty$, for $\mu \in \hat{S}_{K}$, and all $\alpha>0$. In [AM] the Kato class is introduced, which in our notation can be written as $S_{K}=\{\mu \in$ $\left.\hat{S}_{K} ; c(\mu)=0\right\}$. In [Stu] the name Dynkin measure is used for measures in $\hat{S}_{K}$ in the case of the classical Dirichlet form in $L_{2}\left(\mathbb{R}^{n}\right)$. Previously, for this form, the class $S_{K}$ was introduced in [BM] as $G K_{n}$.

We use the slightly larger class $\hat{S}_{K}$ including the quantities $c_{\alpha}(\mu)$ and $c(\mu)$, since it appears that those measures $\mu \in \hat{S}_{K}$ satisfying $c(\mu)<1$ form just the right class of perturbations for which the operator ' $H-\mu$ ' (in a suitable sense) can be defined in all $L_{p}(X, m), 1 \leqslant p \leqslant \infty$. For the case of the classical Dirichlet form on $\mathbb{R}_{n}^{n}$, the Kato class $K_{n}$ (cf. [Si1]) was extended in the analogous way to $\hat{K}_{n}$ in [Vo1].

1.7. REMARKS. (a) For $\mu \in S_{0}, \alpha>0$ there exists $(H+\alpha)^{-1} \mu \in D$ which is uniquely determined by the equation

$$
\int u^{\sim} \mathrm{d} \mu=(b+\alpha)\left[u,(H+\alpha)^{-1} \mu\right] \quad\left(u \in D \cap C_{c}(X)\right) .
$$

For $V \in L_{2}(X, m)_{+}$it follows that $V m \in S_{0}$ and

$$
(H+\alpha)^{-1} V m=(H+\alpha)^{-1} V
$$

for $\alpha>0$, where $(H+\alpha)^{-1}$ is the resolvent of $H$.

(b) For $\mu \in S_{0}$ we have the equivalence

$$
\mu \in \hat{S}_{K} \Leftrightarrow \exists \alpha>0:(H+\alpha)^{-1} \mu \in L_{\infty},
$$

and for $\mu \in \bar{S}_{K^{r}} \cap S_{0}$ the functional $\Phi(\mu, \alpha)$ is generated by $(H+\alpha)^{-1} \mu$.

(c) $\hat{S}_{K} \subset S$. This follows, since $(I I+\alpha)^{-1}\left(C_{c}(X)\right)$ is a dense subspace of $D$ which is contained in $L_{\infty}(m)$ and in $\mathcal{L}_{1}(X, \mu)$ for any $\mu \in \hat{S}_{K}$. Consequently, $D(\mu)$ is dense in $D$ and Theorem 1.5 yields the assertion. (In Theorem 3.1 it will be shown that each $\mu \in \hat{S}_{K^{\prime}}$ is b-bounded.)

1.8. EXAMPLES. (a) By the preceding remarks it is easy to give examples of measures $\mu=V m$ which lie in $S_{0}, \hat{S}_{K}, S_{K}$ with respect to the trivial Dirichlet form $\mathfrak{h}=0$ :

$$
\begin{aligned}
& V m \in S_{0} \Leftrightarrow V \in L_{2}, \\
& V m \in S_{K} \Leftrightarrow V \in L_{\infty} .
\end{aligned}
$$

(In this case $\hat{S}_{K^{*}}=S_{K^{\circ}}$ ) 
(b) A typical example of a measure in $M_{0}$ is given by the following definition: Fix $B \subset X$ and let (cf. [BDM; Def. 2.2, p. 5])

$$
\infty_{B}(A):= \begin{cases}\infty & \text { if } \operatorname{cap}(A \cap B) \neq 0 \\ 0 & \text { otherwise. }\end{cases}
$$

Then $\infty_{B} \in M_{0}$.

(c) For $B \in \mathfrak{B}$, the measure $\infty \chi_{B}$,

$$
\infty \chi_{B}(A)= \begin{cases}\infty & \text { if } m(A \cap B) \neq 0, \\ 0 & \text { otherwise }\end{cases}
$$

and belongs to $M_{0}$.

(d) For the classical Dirichlet form in $L_{2}(\mathbb{R}), \delta \in S_{K} \cap S_{0}$. More generally, for the classical Dirichlet form in $L_{2}\left(\mathbb{R}^{n}\right)$, the surface measure of every 1codimensional compact $C^{\mathbf{l}}$-submanifold of $\mathbb{R}^{d}$ lies in the Kato class; cf. [BEKS; Section 4].

We conclude this section by an approximation result which will serve as one of the steps in the approximation of measure perturbations by functions for a large class of measures, including smooth measures.

This result and the subsequent corollary generalize [AM1; Theorem 2.4]; the proof given there uses probabilistic methods and hence does not carry over to our general context.

1.9. PROPOSITION. Let $\mu \in S_{0}$. Then there exists an increasing sequence $\left(F_{n}\right)$ of closed sets such that $\operatorname{cap}\left(X \backslash F_{n}\right) \rightarrow 0(n \rightarrow \infty), \chi_{F_{n}} \mu \in S_{K}$ for all $n \in \mathbb{N}$.

Proof. (i) We first show $(H+\alpha)^{-1} \mu \rightarrow 0$ in $D$ for $\alpha \rightarrow \infty$. If $u \in D$, then clearly $(H+\alpha)^{-1} u \rightarrow 0$. Further, the family $\left((H+\alpha)^{-1} ; \alpha \geqslant 1\right)$ is bounded in $L\left(D^{*}, D\right)$. Since $D$ is dense in $D^{*}$ we get $(H+\alpha)^{-1} \Phi \rightarrow 0$ in $D$ for all $\Phi \in D^{*}$. Because of $S_{0} \subset D^{*}$ we obtain the assertion.

(ii) We show: For $\varepsilon>0$ there exists a closed set $F \subset X, \operatorname{cap}(X \backslash F)<\varepsilon$ such that $\chi F \mu \in S_{K}$.

For $k \in \mathbb{N}$ we have (cf. [Fu; Lemma 3.1.5])

$$
\begin{aligned}
& \operatorname{cap}\left\{x \in X ;\left((H+\alpha)^{-1} \mu\right)^{\sim}(x)>\frac{1}{k}\right\} \\
& \quad \leqslant k^{2}\left(\mathfrak{h}\left[(H+\alpha)^{-1} \mu\right]+\left\|(H+\alpha)^{-1} \mu\right\|_{2}^{2}\right) .
\end{aligned}
$$

Therefore, (i) implies that there exists an open set $U_{k}$ with $\operatorname{cap}\left(U_{k}\right)<2^{-k} \varepsilon$ and $\alpha_{k}>0$ such that

$$
\left(\left(H+\alpha_{k}\right)^{-1} \mu\right)^{\sim} \leqslant \frac{1}{k} \quad \text { q.e. on } X \backslash U_{k} .
$$


Define $U:=\cup_{k=1}^{\infty} U_{k}, F:=X \backslash U$. Then $\operatorname{cap}(U) \leqslant \varepsilon$ (by [Fu; Lemma 3.1.2]). Further, for $k \in \mathbb{N}$,

$$
\left(\left(H+\alpha_{k}\right)^{-1}\left(\chi_{F} \mu\right)\right)^{\sim} \leqslant\left(\left(H+\alpha_{k}\right)^{-1} \mu\right)^{\sim} \leqslant \frac{1}{k} \quad \text { q.e. on } \operatorname{supp}\left(\chi_{F} \mu\right) \subset F,
$$

hence

$$
\left(\left(H+\alpha_{k}\right)^{-1}\left(\chi_{F} \mu\right)\right)^{\sim} \leqslant \frac{1}{k} \quad\left(\chi_{F} \mu\right)-\text { a.e. }
$$

From [Fu; Lemma 3.2.3] we conclude

$$
\left(\left(H+\alpha_{k}\right)^{-1}\left(\chi_{F} \mu\right)\right)^{\sim} \leqslant \frac{1}{k} \quad m-\text { a.e. }
$$

So we have shown $\chi_{F} \mu \in S_{K}$.

(iii) Choose a null sequence $\left(\varepsilon_{n}\right)$ and choose $F_{n}$ corresponding to $\varepsilon_{n}$ according to (ii), without restriction $\left(F_{n}\right)$ increasing.

1.10. COROLLARY. Let $\mu$ be a Borel measure on $X$. Then the following are equivalent:

(i) $\mu$ is smooth.

(ii) For every compact $K \subset X$ there exists a $K$-nest $\left(F_{n}\right)$ such that $\chi_{F_{n}} \mu \in S_{K}$ for all $n \in \mathbb{N}$.

Proof. Clear from Theorem 1.6 and Proposition 1.9.

\section{Approximation of Measures in $\hat{S}_{K} \cap S_{0}$}

The aim of the present section is to approximate measures $\mu$ by measures $V m$ generated by functions. This will finally allow us to transfer certain properties from operators of the form $H-V$ to operators ' $I T-\mu$ '. The essential step is the following approximation result.

2.1. THEOREM. Let $\mu \in \hat{S}_{K} \cap S_{0}, \alpha>0, \gamma:=c_{\alpha}(\mu)$. Then there exists a sequence $\left(V_{n}\right)$ in $L_{2}(m) \cap L_{\mathrm{co}}(m)_{+}$such that

$$
\begin{aligned}
& c_{\alpha}\left(V_{n}\right) \leqslant \gamma \quad(n \in \mathbb{N}), \\
& \int V_{n}|u|^{2} \mathrm{~d} m \leqslant \gamma\left(\mathrm{h}[u]+\alpha \|\left. u\right|^{2}\right) \quad(n \in \mathbb{N}, u \in D),
\end{aligned}
$$

and $V_{n} \rightarrow \mu$ strongly in $L\left(D, D^{*}\right)$. Further,

$$
\mu[u] \leqslant \gamma\left(\mathfrak{h}[u]+\alpha\|u\|^{2}\right) \quad(u \in D) .
$$


(The strong convergence in $L\left(D, D^{*}\right)$ can also be expressed as follows: For all $u, v \in D$, the sequence $\int u \bar{v} V_{n} \mathrm{~d} m$ converges to $\int u \bar{v} \mathrm{~d} \mu$, and for each $u$ the convergence is uniform for $v$ in the unit ball of $D$.)

The approximation of $\mu$ by functions will be achieved by smoothing with resolvents of $H$ as introduced in Remark 1.7(a). For $n \in \mathbb{N}$ we use the mapping

$$
n(H+n)^{-1}: S_{0} \rightarrow S_{0} .
$$

2.2. LEMMA (cf. [Fu; Lemma 3.2.1, p. 70]). For every $\mu \in S_{0}$, the following statements hold:

(i) $n(H+n)^{-1} \mu \rightarrow \mu$ in $D^{*}$ for $n \rightarrow \infty$.

(ii) $n(H+n)^{-1} \mu \rightarrow \mu$ vaguely for $n \rightarrow \infty$.

(iii) For all $n \in \mathbb{N}, \alpha>0$ :

$$
\left(1-\frac{\alpha}{n}\right)(H+\alpha)^{-1} n(H+n)^{-1} \mu \leqslant(H+\alpha)^{-1} \mu .
$$

Proof. (i) This follows from the fact that $n(H+n)^{-1}$, interpreted suitably on $D^{*}$, converges strongly to the identity.

(ii) For $u \in D \cap C_{c}(X)$, (i) implies $\int u n(H+n)^{-1} \mu \mathrm{d} m \rightarrow \int u \mathrm{~d} \mu$. This carries over to $u \in C_{c}(X)$ by approximation ( $h$ is regular).

(iii) is a consequence of the resolvent equation and the positivity of $(H+\alpha)^{-1}$ for $\alpha>0$.

The following proposition will supply the bounds for the approximations $V_{n}$ in Theorem 2.1.

2.3. PROPOSITION. Let $V \in L_{2}(X, m)_{+}, \alpha>0,(H+\alpha)^{-1} V \in L_{\infty}(X, m)$. Then for all $u \in D \cap C_{c}(X)$ :

$$
\int V|u|^{2} \mathrm{~d} m \leqslant\left\|(H+\alpha)^{-1} V\right\|_{\infty}(\mathfrak{h}+\alpha)[u] .
$$

Proof (cf. [Sil; p. 259]). Without restriction we may assume $V \in L_{\infty}(X, m)$ (if necessary, approximate $V$ from below by bounded functions). Then $\left\|(H+\alpha)^{-1} V\right\|_{\infty}=\left\|(H+\alpha)^{-1} V\right\|_{\infty, \infty}$ (the norm of the operator from $L_{\infty}$ to $\left.L_{\infty}\right)$. By duality $\left\|V(H+\alpha)^{-1}\right\|_{1,1}=\left\|(I+\alpha)^{-1} V\right\|_{\infty, \infty}$. Applying the Stein interpolation theorem, one obtains

$\left\|V^{1 / 2}(H+\alpha)^{-1 / 2}\right\|_{2,2}^{2}=\left\|V^{1 / 2}(H+\alpha)^{-1} V^{1 / 2}\right\|_{2,2} \leqslant\left\|(H+\alpha)^{-1} V\right\|_{\infty, \infty}$,

which implies the assertion. Here we have assumed without restriction (see proof of Theorem 5.1) that $\mathbb{K}=\mathbb{C}$. 
Proof of Theorem 2.1. Let $u_{0}:=(H+\alpha)^{-1} \mu\left(\epsilon L_{\infty}(X, m)\right)$. For $n \in \mathbb{N}$ let $V_{n}:=(n-\alpha)(H+n)^{-1} \mu=\left(1-\frac{\alpha}{n}\right) n(H+n)^{-1} \mu$. From Lemma 2.2 (iii) we conclude

$$
(H+\alpha)^{-1} V_{n}=\left(1-\frac{\alpha}{n}\right)(H+\alpha)^{-1} n(H+n)^{-1} \mu \leqslant(H+\alpha)^{-1} \mu=u_{0},
$$

and therefore

$$
c_{\alpha}\left(V_{n}\right) \leqslant \gamma
$$

From Proposition 2.3 we conclude

$$
\int|u|^{2} V_{n} \mathrm{~d} m \leqslant \gamma(\mathfrak{h}+\alpha)[u] \quad\left(u \in D \cap C_{c}(X)\right),
$$

and this implies, by Lemma 2.2 (ii),

$$
\mu[u]=\int|u|^{2} \mathrm{~d} \mu=\lim _{n \rightarrow \infty} \int|u|^{2} V_{n} \mathrm{~d} m \leqslant \gamma(\mathfrak{h}+\alpha)[u]
$$

for all $u \in D \cap C_{c}(X)$.

Finally we prove the strong convergence $V_{n} \rightarrow \mu$ in $L\left(D, D^{*}\right)$. The previous facts show that the sequence $\left(V_{n} m\right)$ is uniformly bounded in $L\left(D, D^{*}\right)$. It is therefore sufficient to show that $V_{n} u \rightarrow u \mu$ in $D^{*}$, or equivalently,

$$
v_{n}:=(H+\alpha)^{-1}\left(V_{n} u\right) \rightarrow v:=(H+\alpha)^{-1}(u \mu) \text { in } D
$$

for every $u \in D \cap C_{c}(X)_{+}$.

We calculate

$$
\begin{aligned}
(\mathfrak{h}+\alpha)\left[v-v_{n}\right] & =\int\left(v-v_{n}\right) u \mathrm{~d} \mu-\int\left(v-v_{n}\right) u V_{n} \mathrm{~d} m \\
& =(\mathfrak{h}+\alpha)\left[\left(v-v_{n}\right) u,(H+\alpha)^{-1} \mu-(H+\alpha)^{-1} V_{n}\right] .
\end{aligned}
$$

Since $(H+\alpha)^{-1} V_{n} \rightarrow(H+\alpha)^{-1} \mu$ in $D$, by Lemma 2.2 (i) it remains to show that $\left(\left(v-v_{n}\right) u\right)$ is bounded in $D$. From [Fu; Thm. 1.4.2, p. 25] we obtain

$$
\left\|\left(v-v_{n}\right) u\right\|_{\mathfrak{h}} \leqslant\left\|v-v_{n}\right\|_{\infty} \cdot\|u\|_{\mathfrak{h}}+\left\|v-v_{n}\right\|_{\mathfrak{h}} \cdot\|u\|_{\infty} .
$$

The boundedness of the first term follows from the inequalities

$$
\begin{aligned}
& 0 \leqslant(H+\alpha)^{-1}\left(V_{n} u\right) \leqslant \gamma\|u\|_{\infty} \text { for } n \in \mathbb{N}, \\
& 0 \leqslant(H+\alpha)^{-1}(u \mu) \leqslant \gamma\|u\|_{\infty} .
\end{aligned}
$$


The boundedness of the second term is obtained from

$$
\begin{aligned}
(\mathfrak{h}+\alpha)\left[v_{n}\right] & =\int\left[(H+\alpha)^{-1}\left(V_{n} u\right)\right] V_{n} u \mathrm{~d} m \\
& \leqslant\|u\|_{\infty} \gamma \int u V_{n} \mathrm{~d} m \rightarrow\|u\|_{\infty} \gamma \int u \mathrm{~d} \mu,
\end{aligned}
$$

where we have used the vague convergence stated in Lemma 2.2 (ii).

2.4. COROLLARY. Let $\mu \in \hat{S}_{K} \cap S_{0}, c(\mu)<1$. Then, by the definition of $c(\mu)$, there exists $\alpha>0$ such that $\gamma:=c_{\alpha}(\mu)<1$. Let $\left(V_{n}\right)$ be as in Theorem 2.1. Then:

(a) The forms $\mathfrak{h}-\mu, \mathfrak{h}-V_{n}(n \in \mathbb{N})$ are bounded below by $-\gamma \alpha$. Denote by $H_{-\mu}$ the selfadjoint operator corresponding to $b-\mu$. Then we have strong resolvent convergence

$$
H-V_{n} \rightarrow H_{-\mu} \text {. }
$$

(b) The $C_{0}$-semigroups $\left(e^{-t H_{-\mu}} ; t \geqslant 0\right),\left(e^{-t\left(H-V_{n}\right)} ; t \geqslant 0\right)$ on $L_{2}(X, m)$ act also as $C_{0}$-semigroups on $L_{p}(X, m)$ for $p \in[1, \infty)$. Denote by $U_{p,-\mu}(\cdot), U_{p,-V_{n}}(\cdot)$ $(n \in \mathbb{N})$ the respective $C_{0}$-semigroups on $L_{p}(X, m)$. Then, for all $p \in(1, \infty)$, the strong convergence

$$
U_{p,-\mu}(t)=s-\lim _{n \rightarrow \infty} U_{p,-V_{n}}(t)
$$

holds uniformly for $t$ in bounded intervals. There exists constants $M \geqslant 0, \omega \in \mathbb{R}$ depending only on $\alpha, \gamma$ such that

$$
\left\|U_{p,-\mu}(t)\right\|_{p, p} \leqslant M e^{\omega t} \quad(t \geqslant 0, p \in[1, \infty)) .
$$

Finally, for $p \in[1, \infty), f \in L_{p}(X, m)_{+}, t \geqslant 0$ the inequality

$$
U_{p,-\mu}(t) f \geqslant U_{p}(t) f \geqslant 0
$$

holds. (For ' $U_{p}(\cdot)$ ' see the notation introduced subsequently.)

In order to prove part (b) of the corollary we recall the significance of the constant $c_{\alpha}(\cdot)$ in connection with the Miyadera perturbation theorem for $C_{0}$-semigroups (cf. [Vo]).

First we recall that, by the second Beurling-Deny criterion, $e^{-t H}$ is a contraction in all $L_{p}(X, m)(1 \leqslant p \leqslant \infty)$. The induced semigroup will be denoted by $U_{p}(\cdot)$ and its generator by $-H_{p}$, for $p \in[1, \infty)$.

2.5. LEMMA. Let $\alpha>0,0 \leqslant \gamma<\gamma^{\prime}<1$. Then there exists $\alpha^{\prime}>0$ such that for all $V \in L_{\infty}(X, m)_{+}$satisfying $c_{\alpha}(V) \leqslant \gamma$ the following inequality holds:

$$
\int_{0}^{\alpha^{\prime}}\left\|V U_{1}(t) f\right\|_{1} \mathrm{~d} t \leqslant \gamma^{\prime}\|f\|_{1} \quad\left(f \in L_{1}(X, m)\right)
$$


As a consequence $-H_{1}+V$ generates a $C_{0}$-semigroup $U_{1,-V}(\cdot)$, and the constants $M \geqslant 0, \omega \in \mathbb{R}$ in the estimate $\left\|U_{1,-V}(t)\right\|_{i} \leqslant M e^{\omega t}, t \geqslant 0$, depend only on $\alpha, \gamma, \gamma^{\prime}$.

Proof. First we show the equality $c_{\alpha}(V)=\left\|V\left(H_{1}+\alpha\right)^{-1}\right\|_{1,1}$ : For $f \in$ $D \cap C_{c}(X)_{+}$we have

$$
\Phi(V, \alpha) f=\int_{X}\left((I+\alpha)^{-1} f\right)^{\sim} V \mathrm{~d} m=\int_{X} V(H+\alpha)^{-1} f \mathrm{~d} m .
$$

Taking the supremum over those $f$ with $\|f\|_{1} \leqslant 1$ the asserted equality is obtained.

Now inequality (2.1) follows from [Vo1; Proposition 4.7(b)]. The last statement is then a consequence of the Miyadera perturbation theorem as stated in [Vo].

Proof of Corollary 2.4. (a) The lower bound for $\mathfrak{h}-\mu, \mathfrak{h}-V_{n}$ follows immediately from the estimates given in Theorem 2.1 , e.g. for $\mathfrak{h}-\mu$ :

$$
\mathfrak{h}[\mu]-\mu[u] \geqslant \mathfrak{h}[u]-\gamma \mathfrak{h}[u]-\gamma \alpha\|u\|^{2} \geqslant-\gamma \alpha\|u\|^{2} .
$$

Now the strong resolvent convergence $H-V_{n} \rightarrow H_{-\mu}$ is a consequence of Theorem A.1 (Appendix A).

(b) For $p=2$, the assertion is a consequence of (a). Choosing $\gamma^{\prime} \in(\gamma, 1)$ we conclude from Lemma 2.5 that the $C_{0}$-semigroups $U_{1,-V_{n}}(\cdot)$ satisfy a uniform estimate

$$
\left\|U_{1,-V_{n}}(t)\right\|_{1,1} \leqslant M e^{\omega t} \quad(n \in \mathbb{N}, t \geqslant 0) .
$$

Applying duality and interpolation, the same estimate is obtained for $U_{p,-V_{n}}(\cdot)$, for $p \in[1, \infty)$.

The strong resolvent convergence $H-V_{n} \rightarrow H_{-\mu}$ implies the strong convergence

$$
e^{-t H_{-\mu}}=s-\lim _{n \rightarrow \infty} e^{-t\left(H-V_{n}\right)},
$$

uniformly for $t$ in bounded intervals; cf. [Pa; sect. 3.4]. Moreover, the inequality $e^{-t\left(H-V_{n}\right)} f \geqslant e^{-t H} f \geqslant 0$, valid for all $f \in L_{2}(X, m)_{+}, t \geqslant 0, n \in \mathbb{N}$, carries over to the limit.

Let $f \in L_{1} \cap L_{\infty}(X, m), t>0$. Then the sequence $\left(e^{-t\left(H-V_{n}\right)} f\right)_{n \in \mathbb{N}}$ converges to $e^{-t H_{-\mu}} f$ in $L_{2}(X, m)$ and is bounded in $L_{1}(X, m)$ as well as in $L_{\infty}(X, m)$ (the latter by duality). This implies that $e^{-t\left(H-V_{n}\right)} f$ converges to $e^{-t H_{-\mu}} f$ in $L_{p}(X, m)$ for all $p \in(1, \infty)$; this convergence is uniform for $t$ in bounded intervals. For $p=1$ the Fatou lemma implies

$$
\begin{aligned}
\left\|e^{-t H T_{-\mu}} f\right\|_{1} & \leqslant \liminf \left\|e^{-t\left(H-V_{n}\right)} f\right\|_{1} \\
& \leqslant M e^{\omega t}\|f\|_{1} .
\end{aligned}
$$


The convergence shown in the preceding paragraph implies the existence and the strong continuity of $U_{p,-\mu}(\cdot)$, for $p \in(1, \infty)$. For $p=1$, the estimate shown above proves the existence of a one-parameter semigroup $U_{1,-\mu}(\cdot)$. Since $U_{1,-\mu}(t)$ is positivity preserving for all $t \geqslant 0$, the strong continuity follows from [Vo3].

2.6. REMARK. If $\mu, \mu^{\prime} \in \hat{S}_{K} \cap S_{0}, c\left(\mu^{\prime}\right)<1, \mu \leqslant \mu^{\prime}$, then

$$
U_{p,-\mu}(t) f \leqslant U_{p,-\mu^{\prime}}(t) f
$$

for all $p \in[1, \infty], f \in L_{p}(X, m), t \geqslant 0$.

For $p=2$, this follows from Corollary B.3 (Appendix B), and it carries over to general $p$.

\section{Approximation of Measures in $\hat{S}_{K}$}

3.1. THEOREM. Let $\mu \in \hat{S}_{K}$. Then $\mu$ is $\mathfrak{h}$-bounded. More precisely, if $\alpha>0$, $\gamma:=c_{\alpha}(\mu)$, then

$$
\mu[u] \leqslant \gamma\left(\mathfrak{h}[u]+\alpha\|u\|^{2}\right)
$$

Proof. Let $K \subset X$ be compact. By Theorem 1.6 and Remark 1.7(c) there exists a $K$-nest $\left(F_{n}\right)$ such that $\chi F_{n} \mu \in S_{0}$ for all $n \in \mathbb{N}$. The definition of $\hat{S}_{K}$ implies $\chi F_{n} \mu \in \hat{S}_{K}, c_{\alpha}\left(\chi F_{n} \mu\right) \leqslant c_{\alpha}(\mu)=\gamma(n \in \mathbb{N})$. Theorem 2.1 implies

$$
\int_{F_{n}}\left|u^{\sim}\right|^{2} \mathrm{~d} u \leqslant \gamma\left(\mathfrak{h}[u]+\alpha\|u\|^{2}\right) \quad(u \in D, n \in \mathbb{N}) .
$$

From $\mu \in M_{0}$ one obtains

$$
\int_{K}\left|u^{\sim}\right|^{2} \mathrm{~d} \mu=\lim _{n \rightarrow \infty} \int_{F_{n} \cap K}\left|u^{\sim}\right|^{2} \mathrm{~d} \mu \leqslant \gamma\left(\mathfrak{h}[u]+\alpha\|u\|^{2}\right) \quad(u \in D) .
$$

Since $K$ is arbitrary we obtain the desired inequality.

3.2. REMARK. If $\mu \in M_{0}$ is $\mathfrak{h}$-bounded and $\mu(X)<\infty$, then $\mu \in S_{0}$ (clear because of $\left.\int\left|u^{\sim}\right| \mathrm{d} \mu \leqslant \mu[u]^{1 / 2} \mu(X)^{1 / 2}\right)$. In particular, if $\mu \in \hat{S}_{K}$ has compact support, then $\mu \in S_{0}$.

Before we proceed to the main result of this section, we introduce some notation. For $\mu \in \hat{S}_{K}, c(\mu)<1$, Theorem 3.1 implies that $\mathfrak{h}-\mu$ defines a closed form, whose associated selfadjoint operator will be denoted by $H_{-\mu}$. We let

$$
\mathcal{K}:=\{K \subset X ; K \text { compact }\}
$$

directed by inclusion; for $K \in \mathcal{K}$ define

$$
\mu_{K}:=\chi_{K} \mu
$$


3.3. THEOREM. Let $\mu \in \hat{S}_{K}, c(\mu)<1$. There exists $\alpha>0$ such that $\gamma:=$ $c_{\alpha}(\mu)<1$. Then:

(a) $\mathfrak{h}-\mu$ and $\mathfrak{h}-\mu_{k}(K \in \mathcal{K})$ are bounded below by $-\gamma \alpha$. For the associated operators,

$$
H_{-\mu_{K}} \rightarrow H_{-\mu}
$$

in strong resolvent sense.

(b) For all $p \in[1, \infty)$, the semigroup $\left(e^{-t H_{-\mu}} ; t \geqslant 0\right)$ acts also as a $C_{0^{-}}$ semigroup on $L_{p}(m)$, which we denote by $U_{p,-\mu}(\cdot)$;

$$
U_{p,-\mu}(t)=s-\lim _{K \in \mathcal{K}} U_{p,-\mu_{K}}^{J^{\prime}}(t)
$$

uniformly for $t$ in bounded subsets of $[0, \infty)$.

(c) There are constants $M \geqslant 0, \omega \in \mathbb{R}$ depending only on $\alpha, \gamma$ such that

$$
\left\|U_{p,-\mu}(t)\right\| \leqslant M e^{\omega t}
$$

for all $t \geqslant 0, p \in[1, \infty)$.

Proof. (a) The semiboundedness follows from Theorem 3.1. The strong resolvent convergence can be seen with a proof as in [RS; supplement to VIII.7, pp. $372-377]$. One only has to realize that a bounded monotone net of selfadjoint operators is strongly convergent', this is proved as in [RN; proof of 'Satz in Nr. 104'].

(b) The strong convergence

$$
U_{2,-\mu}(t)=s-\lim _{I \in \in K} U_{2,-\mu_{K}}(t)
$$

follows from the strong resolvent convergence $H_{-\mu_{K^{*}}} \rightarrow H_{-\mu_{k}}$ with the same proof as for sequences; cf. [Pa; chap. 3, Thm. 4.2]. From Corollary 2.4 and Remark 3.2 we infer that $U_{p,-\mu_{K}}(\cdot)$ is a $C_{0}$-semigroup for $K \in \mathcal{K}$, and the bounds $\left\|U_{p_{1}-\mu_{K}}(t)\right\| \leqslant$ $M e^{\omega t}$ in Corollary 2.4 are independent of $K$. Fix $p \in[1, \infty), f \in L_{p}(X, m)_{+}$, $t \geqslant 0$. Remark 2.6 implies that $\left(U_{p,-\mu_{K}}(t) f\right)_{K \in \mathcal{K}}$ is monotone increasing in $L_{p}(X, m)$. Since it is bounded, it is convergent;

$$
U_{p,-\mu}(t) f:=\lim _{K_{K} \in \mathcal{K}} U_{p,-\mu_{\mathcal{K}}}(t) f .
$$

We conclude that $U_{p,-\mu}(t)=e^{-t H_{-\mu}}$ on $L_{2} \cap L_{p}(X, m), U_{p,-\mu}(\cdot)$ is a oneparameter semigroup, and $U_{p,-\mu}(t) f \geqslant U_{p}(t) f \geqslant 0$ for $f \in L_{p}(X, m)_{+}$.

This implies that $U_{p,-\mu}(\cdot)$ is strongly continuous for all $p \in[1, \infty)$; cf. [Vo2; Proposition A.1]. Hence the uniform convergence follows from the Dini theorem.

(c) follows from (b). 
3.4. REMARK. For the case of the classical Dirichlet form in $L_{2}\left(\mathbb{R}^{n}\right)$, there is a different possibility to approximate measures in $\hat{S}_{K}$, and even more generally, measures which are $\mathfrak{h}$-bounded. Namely, convolution with a $\delta$-sequence provides an appropriate approximation. Since this has been carried out in [HS] we will not discuss it further.

Corollary 2.4 and Theorem 3.3 together show that we have a two-step approximation for measures in $\hat{S}_{K^{-}}$. First they are approximated from below by

$$
\mu_{K}, K \in \mathcal{K}
$$

and since each $\mu_{K}$ belongs to $\hat{S}_{K} \cap S_{0}$, it can be approximated by functions $V_{K, n}$. The point is that we can control the constants $c_{\alpha}$ during this process. For further reference we state this consequence explicitly.

3.5. THEOREM. Let $\mu \in \hat{S}_{K}, \alpha>0, \gamma:=c_{\alpha}(\mu)<1$. Then there exists a net $\left(V_{\iota}\right)_{\iota \in I}$ of functions $V_{\iota} \in L_{2} \cap L_{\infty}(X, m)_{+}$such that

(i) $c_{\alpha}\left(V_{t}\right) \leqslant \gamma \quad(\quad \in I)$,

(ii) $H-V_{\iota} \rightarrow I_{-\mu}$ is strong resolvent sense, (iii) $e^{-t\left(H-V_{1}\right)} \rightarrow e^{-t H_{-\mu}}$ strongly, for all $t \geqslant 0$.

If $(X, m)$ is such that $L_{2}(X, m)$ is separable, then the net $\left(V_{\iota}\right)$ can be chosen as a sequence.

The last statement of the theorem follows from the fact that, due to the separability, bounded sets of operators are metrizable for the strong operator topology.

With this theorem at hand, one can carry over certain results known for $H-V$ to perturbations of the form $H_{-\mu}$, namely those which rely on the constants $c_{\alpha}$. We have already used this strategy in the proof of the relative boundedness of measures in the extended Kato class, Theorem 3.1. A further demonstration of this method will be given in Section 5 , where we prove $L_{p}-L_{q}$ continuity of the semigroups.

\section{Perturbation by General Measures}

4.1. THEOREM. Let $\mu_{+} \in M_{0}, \mu_{-} \in \hat{S}_{木_{s}}, c\left(\mu_{-}\right)<1$.

(a) The closure of $D\left(\mu_{+}\right)$(cf. Section I) in $L_{2}(X, m)$ is of the form $L_{2}(Y, m)$ with a locally measurable set $Y \subset X$.

(b) Denote by $H_{\mu}=H_{-\mu_{-}+\mu_{+}}$the selfadjoint operator in $L_{2}(Y, m)$ associated with the form $\mathfrak{h}-\mu_{-}+\mu_{+}$. Then

$$
0 \leqslant e^{-t H_{\mu}} f \leqslant e^{-t H_{-\mu_{-}-} f}
$$

for all $f \in L_{2}(Y, m)_{+}, t \geqslant 0$. 
Proof. (a) See the remarks following Proposition B.1 (Appendix B).

(b) Clearly, $D\left(\mu_{+}\right)$is an order ideal in $D$. We want to apply Corollary B.3 with unperturbed form $\mathfrak{h}-\mu_{-}+\mathfrak{c}$ (in Corollary B.3: $\mathfrak{b}$ ) and perturbed form $\mathfrak{b}-\mu_{-}+c+\mu_{+}$ (in Corollary B.3: $\mathfrak{k}$ ), where $c \geqslant 0$ is chosen such that $\mathfrak{b}-\mu_{-}+c \geqslant 0$. (Note that, in general, $\mathfrak{h}-\mu_{-}+c$ will not be a Dirichlet form; the conditions of the first Beurling-Deny criterion, however, are still satisfied.)

Now it is easy to see that all the hypotheses of Corollary B.3 are satisfied, and the conclusion yields (b).

4.2. COROLLARY. Let $\mu_{+}, \mu_{-}, Y, H_{\mu}$ be as in Theorem 4.1. Then the semigroup $\left(e^{-t H_{\mu}} ; t \geqslant 0\right)$ acts as a positive $C_{0}$-semigroup $U_{p, \mu}(\cdot)$ on $L_{p}(Y, m)$, for all $p \in[1, \infty)$, and the bounds for the operators in $L_{p}(Y, m)$ are the same as for $\left(e^{-t H_{-\mu_{-}}} ; t \geqslant 0\right)$, i.e. the bounds given in Theorem 3.3.

Proof. This is obvious from the inequality given in Theorem 4.1. The strong continuity of $U_{p, \mu}(\cdot)$ follows from [Vo3].

At that point, let us compare the scope of measures treated above with the one treated in $[\mathrm{AM}]$. Since we want to study those $\mu$, for which the semigroup still acts in all $L_{p}$-spaces, the assumption $\mu_{-} \in \hat{S}_{K^{\prime}}$ cannot be weakened substantially. In [AM] more general $\mu_{-}$are allowed, since different properties of ' $H-\mu$ ' are investigated. The standard assumption in [AM] concerning the positive part is $\mu_{+} \in S$, which is certainly more restrictive than our assumption $\mu_{+} \in M_{0}$, the latter being minimal, if one wants to use Dirichlet forms or the Feynman-Kac formula.

For the classical Dirichlet form on $\mathbb{R}^{d}$, the class $M_{0}$ has been recognized as 'the right one' for positive perturbations of $-\Delta$ at least in [BDM], where the connections between probabilistic and variational notions are studied; see also [Stu] for a detailed account concerning the associated additive functionals.

4.3. NOTATION. Let $\mu_{+}, \mu_{-}, Y$ etc. be as in Theorem 4.1. The generator of the $C_{0}$-semigroup $U_{p, \mu}(\cdot)$ on $L_{p}(Y, m)$ (see Theorem 4.2 ) will be denoted by $-H_{p, \mu}$. Further, $P$ will denote the projection $P: L_{p}(X, m) \rightarrow L_{p}(Y, m), P f:=\chi_{Y} f$.

We note the following monotonicity property.

4.4. REMARK. Let $\mu_{+}, \mu_{+}^{\prime} \in M_{0}, \mu_{+} \leqslant \mu_{+}^{\prime}$, and $Y^{\prime} \subset Y \subset X$ the corresponding sets; $\overline{D\left(\mu_{+}^{\prime}\right)}=L_{2}\left(Y^{\prime}, m\right), \overline{D\left(\mu_{+}\right)}=L_{2}(Y, m)$. Let $\mu_{-}, \mu_{-}^{\prime} \in \hat{S}_{K}, c\left(\mu_{-}\right)<$ $1, \mu_{-}^{\prime} \leqslant \mu_{-}$. Then $D\left(\mathfrak{h}-\mu_{-}^{\prime}+\mu_{+}^{\prime}\right)=D\left(\mu_{+}^{\prime}\right)$ is an ideal in $D\left(\mu_{+}\right)=D\left(h-\mu_{-}+\right.$ $\left.\mu_{+}\right)$

$$
\left(\mathfrak{h}-\mu_{-}+\mu_{+}\right)[u, v] \leqslant\left(\mathfrak{h}-\mu_{-}^{\prime}+\mu_{+}^{\prime}\right)[u, v]
$$


for all $u, v \in D\left(\mu_{+}^{i}\right)_{+}$, and therefore Corollary B.3 implies

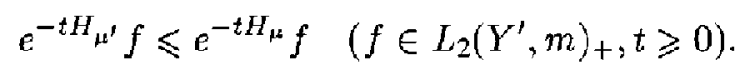

This monotonicity obviously carries over to the induced $L_{\mathfrak{p}}$-semigroups.

Next we present an application of a monotone convergence theorem for forms to our situation.

4.5. THEOREM. Let $\mu_{-} \in \hat{S}_{K}, c\left(\mu_{-}\right)<1$. Let $\left(\mu_{\iota}\right)_{l \in I}$ be an increasing net in $M_{0}, \mu_{+} \in M_{0}$, and $\mu_{+}=\sup _{\iota \in I} \mu_{\iota}$ in the sense that

$$
\sup _{\iota \in I} \mu_{\imath}[u]=\mu_{+}[u] \quad(u \in D)
$$

(where the form $\mu_{+}$takes the value $+\infty$ if $u$ does not belong to the domain). Then, for $1 \leqslant p<\infty, f \in L_{p}(X, m), t>0$, there is convergence

$$
U_{p,-\mu_{-}+\mu_{t}}(t) P_{t} f \rightarrow U_{p,-\mu_{-}+\mu_{+}}(t) P f
$$

(where $P_{\downarrow}$ denotes the canonical projection of Notation 4.3, corresponding to $\left.\mu_{-}, \mu_{i}\right)$. For $f \in L_{p}(Y, m)$, this convergence is uniform for $t$ in compact intervals of $(0, \infty)$.

Proof. (i) Fix $1 \leqslant p<\infty$, for the moment. Then, for $\iota, \kappa \in I, \iota \leqslant \kappa$, Remark 4.4 implies $U_{p,-\mu_{-}+\mu_{l}}(t) P_{\iota} \geqslant U_{p,-\mu_{-}+\mu_{\kappa}}(t) P_{\kappa}(t \geqslant 0)$. Therefore, for $t>0, s-\lim _{\iota \in I} U_{p,-\mu_{-}+\mu_{\iota}}(l) P_{\iota}=: V_{p}(l)$ exists, and $V_{p}(\cdot)$ has the semigroup property $V_{p}(t+s)=V_{p}(t) V_{p}(s)(t, s>0)$. It was shown in [AB, Corollary 3.3] that $V_{p}(\cdot)$ is strongly continuous, that $V_{p}(0):=s-\lim _{t \rightarrow 0} V_{p}(t)$ exists and is a band projection, i.e., $R\left(V_{p}(0)\right)=L_{p}(Z, m)$ for suitable $Z \subset X$, and $V_{p}(0) f=$ $\chi_{Z} f$. In particular, $V_{p}(\cdot)$ is a $C_{0}$-semigroup on $L_{p}(Z, m)$. Dini's theorem implies $U_{p,-\mu_{-}+\mu_{L}}(t) f \rightarrow V_{p}(t) f$ uniformly for $t$ in compact subsets of $(0, \infty)$, for $f \in$ $L_{p}(Z, m)$.

Let $1 \leqslant q<\infty$. Then there exist $V_{q}(\cdot)$ and $Z_{q}$ as above. Clearly, $V_{p}(t)=V_{q}(t)$ on $L_{p} \cap L_{q}(X, m)$, and this implies that $Z=Z_{q}$ is independent of $q$.

(ii) It remains to see that $V_{2}(\cdot)$ coincides with the semigroup associated with the form $h-\mu_{-}+\mu_{+}$. This is shown by a standard method (cf. [Da; Chapter 4 , Theorem 4.3.2]): Let $-S$ be the generator of the $C_{0}$-semigroup $V_{2}(\cdot)$ on $L_{2}(Z, m)$, and $\mathfrak{k}$ the form associated with $S$. Then

$$
\mathfrak{h}-\mu_{-}+\mu_{\iota} \leqslant \mathfrak{l} \leqslant \mathfrak{h}-\mu_{-}+\mu_{+} \quad(\iota \in I),
$$

and this implies $\mathfrak{k}=\mathfrak{h}-\mu_{-}+\mu_{+}$.

Next we illustrate what distinguishes smooth measures from other measures in $M_{0}$, from the point of view of the associated semigroups. The characterization of 
smoothness will be analogous to the definition of 'regular' for absorption rates, given in [Vol; Definition 2.12].

4.6. PROPOSITION. Let $\mu \in M_{0}$. Then the following are equivalent:

(i) $\mu \in S$;

(ii) $U_{p}(t)=s-\lim _{\eta \rightarrow 0+} U_{p, \eta \mu}(t)$ for some (all) $p \in[1, \infty)$ and all $t \geqslant 0$.

The proof is sufficiently analogous to that of [Vol; Proposition 5.8(b)] to be omitted.

4.7. EXAMPLE. Let us now reconsider two of the examples introduced in 1.8 . For $B \subset X, B \subset \mathcal{B}$,

$$
\begin{array}{ll}
D\left(\mathfrak{h}+\infty \chi_{B}\right)=\{f \in D ; f=0 & m \text {-a.e. on } B\} \\
D\left(\mathfrak{h}+\infty_{B}\right)=\left\{f \in D_{;} f^{\sim}=0\right. & \text { q.e. on } B\} .
\end{array}
$$

If we specialize to the classical Dirichlet form on $\mathbb{R}^{d}$, and $B=\bar{B}$, the latter domain equals $W_{2,0}^{1}\left(\mathbb{R}^{d} \backslash B\right)$ (= the closure of $C_{c}^{\infty}\left(\mathbb{R}^{d} \backslash B\right)$ in $W_{2}^{1}\left(\mathbb{R}^{d}\right)$ ). If $B=B_{0} \cup B_{1}$ with disjoint closed sets such that $B_{0}$ is of Lebesgue measure zero, but not of capacity zero, then clearly

$$
D\left(\mathfrak{h}+\infty \chi_{B}\right)=D\left(\mathfrak{h}+\infty \chi_{B_{1}}\right) \supset W_{2,0}^{1}\left(\mathbb{R}^{d} \backslash B_{1}\right) \supsetneqq W_{2,0}^{1}\left(\mathbb{R}^{d} \backslash B\right),
$$

so that $\mathfrak{h}+\infty \chi_{B} \neq \mathfrak{h}+\infty_{B}$.

In fact, this can happen in a more interesting way (as shown in [Sto] where a detailed study of related questions is given): Using Example 1.17 in [He], one can find a compact $K$ such that $K=\bar{K}$ but nevertheless $W_{2,0}^{1}\left(\mathbb{R}^{d} \backslash K\right) \neq\{f \in$ $W_{2}^{\prime} ; f=0$ a.e. on $\left.K\right\}$. This shows that a conjecture in [Si; p. 384] is false. At this point the first named author would like to express his gratitude to Prof. Hedberg, who kindly pointed out [He; Example 1.17] to him.

\section{5. $L_{p}-L_{q}$-Smoothing}

In this section we show that if $H$ has the additional property that $e^{-t H}$ maps $L_{p}$ to $L_{q}$ for $1 \leqslant p \leqslant q \leqslant \infty, t>0$ then the perturbed semigroup has the same property. For the case of $H=-\frac{1}{2} \Delta$ on $\mathbb{R}^{d}$ this is well-established; cf. [Si1], [Dev], [Vo1] for the case of measures of the form $V d x$, [BM] for measures in the Kato class. The proofs in the mentioned papers do not carry over to the present context. We denote by $\|\cdot\|_{p, q}$ the norm in $L\left(L_{p}, L_{q}\right)$.

\subsection{THEOREM. Let $\mu_{+}, \mu_{-}, Y, H_{\mu}$ be as in Theorem 4.1.}

(a) Assume that, for all $t>0$, the operator $e^{-t H}$ is continuous as an operator from $L_{1}(m)$ to $L_{\infty}(m)$. Then $e^{-t H_{\mu}}$ is continuous from $L_{1}(m)$ to $L_{\infty}(m)$, 
for all $t>0$. (And as a consequence of the Riesz-Thorin convexity theorem, $e^{-t H_{\mu}}$ is continuous from $L_{p}(m)$ to $L_{q}(m)$, for $\left.1 \leqslant p \leqslant q \leqslant \infty, t>0\right)$.

(b) Assume, more specifically, that there are constants $C \geqslant 0, d \geqslant 0$ such that, for $1 \leqslant p \leqslant q \leqslant \infty, 0<t \leqslant 1$, the estimate

$$
\left\|e^{-t H}\right\|_{p, q} \leqslant C t^{-\frac{1}{2} d\left(\frac{1}{p}-\frac{1}{q}\right)}
$$

holds. Then there exists $C^{\prime} \geqslant 0$ such that for all $1 \leqslant p \leqslant q \leqslant \infty, 0<t \leqslant 1$ we have

$$
\left\|e^{-t H_{\mu}}\right\|_{p, q} \leqslant C^{\prime} t^{-\frac{1}{2} d\left(\frac{1}{p}-\frac{1}{q}\right)}
$$

Proof. In view of the estimate given in Theorem $4.1(\mathrm{~b})$ it is sufficient to treat the case $\mu_{+}=0$.

Further, in view of Theorem 3.5 it is sufficient to treat the case $\mu_{-}=V d m$, with $V \in L_{\infty}(m)$, if the estimates for the $\|\cdot\|_{p, q}$-norms only depend on the constants $\alpha>0,0 \leqslant \gamma<1$ with $c_{\alpha}(V) \leqslant \gamma$, given in Theorem 3.5 .

(a) For $t>0$ we define

$$
C_{t}:=\left\|e^{-t H}\right\|_{1, \infty}
$$

Choose $\gamma^{\prime}, \gamma<\gamma^{\prime}<1, \kappa>1$ such that $\kappa \gamma^{\prime}<1$, and - for later convenience such that the exponent $k$ conjugate to $\kappa$ is an integer: $\frac{1}{\kappa}+\frac{1}{k}=1$ with $k \in \mathbb{N}$. Then $-H_{1}+\kappa V$ generates a $C_{0}$-semigroup on $L_{1}(m)$, and there are constants $M \geqslant 0, \omega \in \mathbb{R}$ depending only on $\alpha, \kappa \gamma, \kappa \gamma^{\prime}$ such that

$$
\left\|e^{-t\left(H_{1}-\kappa V\right)}\right\|_{1,1} \leqslant M e^{\omega t} \quad(t \geqslant 0)
$$

see Lemma 2.5 .

Since we shall use complex interpolation, we need the field of scalars to be the complex numbers. Thus, if $\mathbb{K}=\mathbb{R}$, we complexify the $L_{p}$-spaces as well as the operators. Note that complexifying does not change the norm of operators from $L_{p}$ to $L_{q}$, for $1 \leqslant p \leqslant q \leqslant \infty$; cf. [FIP].

For $z \in \Sigma:=\{z \in \mathbb{C} ; 0 \leqslant \operatorname{Re} z \leqslant 1\},-H+z \kappa V$ is the generator of a $C_{0}$-semigroup in $L_{2}(m)$. For fixed $t>0$, define

$$
F(z):=e^{-t(H-z \kappa V)} \in L\left(L_{2}(m)\right) .
$$

Then $F$ is continuous on $\Sigma$ and analytic in the interior $\stackrel{s}{\Sigma}$ Let $0 \leqslant r \leqslant 1, s \in \mathbb{R}$. Then, for $f \in L_{2}(m), n \in \mathbb{N}$ we obtain

$$
\left|\left(e^{\frac{t}{n} i s \kappa V} e^{-\frac{t}{n}(H-r \kappa V)}\right)^{n} f\right| \leqslant e^{-t(H-\tau \kappa V)}|f| .
$$

For $n \rightarrow \infty$, the Trotter product formula (cf. [Da; Theorem 3.30]) yields

$$
\left|e^{-t(H-(r+i s) \kappa V)} f\right| \leqslant e^{-t(H-r \kappa V)}|f| .
$$


This shows that $F$ is also bounded on $\Sigma$. It further shows that, for $z \in \Sigma$ with $\operatorname{Re} z=0$,

$$
\|F(z)\|_{1, \infty} \leqslant\|F(0)\|_{1, \infty}=C_{l}
$$

and, for $z \in \Sigma$ with $\operatorname{Re} z=1$,

$$
\|F(z)\|_{1,1} \leqslant\|F(1)\|_{1,1} \leqslant M e^{\omega t} .
$$

Now, the Stein interpolation theorem (see [Ste; p. 69]) implies

$$
\|F(s)\|_{1, p_{s}} \leqslant C_{t}^{1-s}\left(M e^{\omega t}\right)^{s},
$$

where $\frac{1}{p_{s}}=s\left(=\frac{1-s}{\infty}+\frac{s}{1}\right)$, i.e., $p_{s}=\frac{1}{s}$. In particular, for $s=\frac{1}{\kappa}$, we obtain

$$
\left\|e^{-t(H-V)}\right\|_{1, \kappa} \leqslant C_{t}^{\prime}
$$

with $C_{t}^{\prime}:=C_{t}^{\frac{1}{k}} M^{\frac{1}{\kappa}} e^{t \omega \frac{1}{\kappa}} ;$ recall $\frac{1}{k}=1-\frac{1}{\kappa}$.

Duality together with the Riesz-Thorin convexity theorem implies

$$
\|\left. e^{-t(H-V)}\right|_{p, q} \leqslant C_{t}^{\prime}
$$

whenever $1 \leqslant p<q \leqslant \infty, \frac{1}{p}-\frac{1}{q}=\frac{1}{k}$. Choosing $p_{0}=1<p_{1}<\cdots<p_{k}=\infty$ such that $\frac{1}{p_{j-1}}-\frac{1}{p_{j}}=\frac{1}{k}(j=1, \ldots, k)$, we obtain

$$
\left\|e^{-t(H-V)}\right\|_{1, \infty} \leqslant \prod_{j=1}^{k}\left\|e^{-\frac{t}{k}(H-V)}\right\|_{p_{j-1}, p_{j}} \leqslant\left(C_{\frac{t}{k}}^{\prime}\right)^{k}=C_{\frac{t}{k}} M^{k-1} e^{t \omega(k-1)} .
$$

(b) The Riesz-Thorin convexity theorem implies that it is sufficient to show the desired estimate for the case $p=1, q=\infty$.

With the notation of part (a) we have $C_{t} \leqslant C t^{-\frac{1}{2} d}, C_{\frac{t}{k}} \leqslant C k^{\frac{d}{2}} t^{-\frac{d}{2}}$.

5.2. REMARK. The quantity $d$ occuring in Theorem 5.1 (b) is known as the (local) dimension of the diffusion semigroup $\left(e^{-t H} ; t \geqslant 0\right)$; cf. [Co], [Va; Definition, p. 241] for the case $p=1, q=\infty$.

\section{Holomorphy of $L_{1}$-Semigroups}

Let $\mu_{+} \in M_{0}, \mu_{-} \in \hat{S}_{K}, c\left(\mu_{-}\right)<1$, and $Y, H_{\mu}$ be as in Theorem 4.1. Then it follows from the Stein interpolation theorem that, for $1<p<\infty$, the semigroups $U_{p, \mu}(\cdot)$ are holomorphic of angle $\frac{\pi}{2}\left(1-\left|\frac{2}{p}-1\right|\right)$; this is shown as in the usual proof for symmetric diffusion semigroups; see [Ste; Chap. III, sec. 2]. 
In this section we study holomorphy in $L_{1}$, provided the unperturbed semigroup is holomorphic. This investigation has its roots in Kato's paper [Ka1] and moreover was stimulated by the results of Arendt and Batty $[\mathrm{AB}]$.

Thus, in the present section, we assume $\mathbb{K}=\mathbb{C}$, and we assume that $U_{1}(\cdot)$ extends to a holomorphic semigroup of some angle. This assumption is equivalent to requiring that there exist $r \geqslant 0, c \geqslant 1$ such that $\{\lambda \in \mathbb{C}: \operatorname{Re} \lambda \geqslant 0,|\lambda|>r\} \subset$ $\rho\left(H_{1}\right)$,

$$
\left\|\left(H_{1}+\lambda\right)^{-1}\right\| \leqslant \frac{c}{|\lambda|} \quad(\operatorname{Re} \lambda \geqslant 0,|\lambda|>r)
$$

cf. [Na; A-II, Theorem 1.14].

6.1. THEOREM. Let $\mu_{-} \in \hat{S}_{K}, c\left(\mu_{-}\right)<1, \mu_{+} \in S$. Then $U_{1,-\mu_{-}+\mu_{+}}(\cdot)$, the semigroup generated by $H_{1,-\mu_{-}+\mu_{+}}$, is holomorphic.

Proof. (i) First we shall treat the case $\mu_{+}=0$ (cf. [Ka1; proof of Lemma 13]).

There exists $\alpha>0$ such that $\gamma:=c_{\alpha}\left(\mu_{-}\right)<1$. Let $V \in L_{\infty}(m)_{+}, c_{\alpha}(V) \leqslant \gamma$, i.e., $\left\|V\left(H_{1}+\alpha\right)^{-1}\right\|_{1,1} \leqslant \gamma$. For $\lambda \in \mathbb{C}$ with $\operatorname{Re} \lambda \geqslant \alpha$, we then have

$$
\begin{aligned}
\left\|V\left(H_{1}+\lambda\right)^{-1}\right\|_{1,1} & \leqslant\left\|V\left(H_{1}+\operatorname{Re} \lambda\right)^{-1}\right\|_{1,1} \\
& \leqslant\left\|V\left(H_{1}+\alpha\right)^{-1}\right\|_{1,1} \leqslant \gamma .
\end{aligned}
$$

Therefore, if additionally $|\lambda|>r$, the Neumann series

$$
\left(\left(H_{1}-V\right)+\lambda\right)^{-1}=\left(H_{1}+\lambda\right)^{-1} \sum_{j=0}^{\infty}\left(V\left(H_{1}+\lambda\right)^{-1}\right)^{j}
$$

converges, and

$$
\left\|\left(\left(H_{1}-V\right)+\lambda\right)^{-1}\right\| \leqslant\left\|\left(H_{1}+\lambda\right)^{-1}\right\| \sum_{j=0}^{\infty} \gamma^{j} \leqslant \frac{c}{1-\gamma} \frac{1}{|\lambda|} .
$$

Next, let $\tilde{\mu} \in \hat{S}_{K} \cap S_{0}, c_{\alpha}(\tilde{\mu}) \leqslant \gamma$. By Theorem 2.1 there exists a sequence $\left(V_{n}\right)$ in $L_{\infty}(m)_{+}$such that $c_{\alpha}\left(V_{n}\right) \leqslant \gamma(n \in \mathbb{N})$, and $H_{2}-V_{n} \rightarrow H_{2,-\tilde{\mu}}$ in strong resolvent sense, by Corollary 2.4 . From $H_{2}-V_{n} \geqslant-\gamma \alpha>-\alpha$ we obtain

$$
\left\|\left(\left(H_{2}-V_{n}\right)+\lambda\right)^{-1}\right\|_{2,2} \leqslant \frac{1}{\operatorname{Re} \lambda-\gamma \alpha}
$$

for all $n \in \mathbb{N}, \lambda \in \mathbb{C}$ with $\operatorname{Re} \lambda \geqslant \alpha$, and therefore $\left(\left(H_{2}-V_{n}\right)+\lambda\right)^{-1} \rightarrow$ $\left(H_{2,-\bar{\mu}}+\lambda\right)^{-1}$ strongly. For $f \in L_{1} \cap L_{2}(X, m), \lambda \in \mathbb{C}$ with $\operatorname{Re} \lambda \geqslant \alpha,|\lambda|>r$, these facts imply, by Fatou's lemma,

$$
\left\|\left(H_{2,-\bar{\mu}}+\lambda\right)^{-1} f\right\|_{1} \leqslant \liminf _{n \rightarrow \infty}\left\|\left(H_{2}-V_{n}+\lambda\right)^{-1} f\right\|_{1} .
$$


From the representation of resolvents of the generator by the Laplace transform of the semigroup one obtains

$$
\left(H_{2,-\tilde{\mu}}+\lambda\right)^{-1} f=\left(H_{1,-\ddot{\mu}}+\lambda\right)^{-1} f,
$$

and this leads to the inequality

$$
\left\|\left(H_{1,-\tilde{\mu}}+\lambda\right)^{-1}\right\|_{1,1} \leqslant \frac{c}{1-\gamma} \frac{1}{|\lambda|} \quad(|\lambda|>r, \operatorname{Re} \lambda \geqslant \alpha) .
$$

Finally, the approximation procedure of Theorem 3.3 shows that this inequality carries over to $H_{1,-\mu_{-}}$. (Note that in this step one has strong convergence of $L_{1}$ operators at one's disposal, which makes things easier than in the previous step.) The above estimate can be weakened to

$$
\left\|\left(H_{1,-\mu_{-}}+\lambda\right)^{-1}\right\|_{1,1} \leqslant \frac{c}{1-\gamma} \frac{1}{|\lambda-\alpha|} \quad(|\lambda-\alpha|>r, \operatorname{Re} \lambda \geqslant \alpha)
$$

which shows that $-H_{1,-\mu_{-}}-\alpha$ generates a holomorphic semigroup.

(ii) For the general case, let $\mu_{-}, \alpha, \gamma$ be as in (i).

First, let $V \in L_{\infty}(m)_{+}$. It is shown in [AB; proof of Theorem 6.1], that then

$$
\left\|\left(H_{1}+V+\lambda\right)^{-1}\right\|_{1,1} \leqslant \frac{2 c}{|\lambda|} \quad(\operatorname{Re} \lambda \geqslant 0,|\lambda|>r) .
$$

Note that $\mathrm{b}+V$ is again a Dirichlet form, and step (i) can be applied to this Dirichlet form and yields

$$
\left\|\left(H_{1,-\mu_{-}}+V+\lambda\right)^{-1}\right\|_{1,1} \leqslant \frac{2 c}{1-\gamma} \frac{1}{|\lambda-\alpha|} \quad(|\lambda-\alpha|>r, \operatorname{Re} \lambda \geqslant \alpha) .
$$

Next, suppose $\tilde{\mu} \in \hat{S}_{K} \cap S_{0}$. By Theorem 2.1 , there exists a sequence $\left(V_{n}\right)$ in $L_{\infty}(m)_{+}, c_{\alpha}\left(V_{n}\right) \leqslant c_{\alpha}(\tilde{\mu})=: \tilde{\gamma}(n \in N)$, and $V_{n} \rightarrow \tilde{\mu}$ strongly in $L\left(D, D^{*}\right)$. Therefore Theorem A.1 implies

$$
H_{2,-\mu_{-}}+V_{n} \rightarrow H_{2,-\mu_{-}+\bar{\mu}}
$$

in strong resolvent sense. Arguments as in part (i) of the proof yield the estimate

$$
\left\|\left(H_{1,-\mu-+\tilde{\mu}}+\lambda\right)^{-1}\right\|_{1,1} \leqslant \frac{2 c}{1-\gamma} \frac{1}{|\lambda-\alpha|} \quad(|\lambda-\alpha|>r, \operatorname{Re} \lambda \geqslant \alpha) .
$$

(Note that, again, we do not have strong resolvent convergence of the $L_{1}$-operators at our disposal.)

The system

$$
\mathcal{K}:=\left\{K \subset X ; K \text { compact, } \chi_{K} \mu_{+} \in \tilde{S}_{K} \cup S_{0}\right\}
$$


is directed by inclusion. We have

$$
\mathfrak{h}-\mu_{-}+\mu_{+}=\sup _{\kappa^{\prime} \in \mathcal{K}}\left(\mathfrak{h}-\mu_{-}+\chi_{K} \mu_{+}\right) \text {. }
$$

This is a consequence of Corollary 1.10: For $u \in D\left(\mu_{+}\right)$and compact $K^{\prime} \subset X$ there exists a sequence $\left(K_{n}\right)$ in $\mathcal{K}, K_{1} \subset K_{2} \subset \cdots \subset K^{\prime}$, such that $\operatorname{cap}\left(K^{\prime} \backslash \bigcup_{n \in \mathbb{N}} K_{n}\right)=0$. This implies

$$
\begin{aligned}
& \mu_{+}\left(K^{\sim} \backslash \bigcup_{n \in \mathbb{N}} K_{n}\right)=0 \\
& \int_{K_{n}^{\prime}}\left|u^{\sim}\right|^{2} \mathrm{~d} \mu_{+} \rightarrow \int_{K^{\prime}}\left|u^{\sim}\right|^{2} \mathrm{~d} \mu_{+} .
\end{aligned}
$$

From $\int\left|u^{\sim}\right|^{2} \mathrm{~d} \mu_{+}=\sup \left\{\int_{K^{\prime}}\left|u^{\sim}\right|^{2} \mathrm{~d} \mu_{+} ; K^{\prime} \subset X\right.$ compact $\}$ the asserted equality follows.

Now Theorem 4.5 implies strong resolvent convergence

$$
H_{1,-\mu_{-}+\chi_{K} \mu_{+}}^{\underset{K \in K}{\longrightarrow}} H_{1,-\mu_{-}+\mu_{+}} \cdot
$$

This, in turn, implies that the previous estimate carries over,

$$
\left\|\left(H_{1,-\mu_{-}+\mu_{+}}+\lambda\right)^{-1}\right\|_{1,1} \leqslant \frac{2 c}{1-\gamma} \frac{1}{|\lambda-\alpha|} \quad(|\lambda-\alpha|>r, \operatorname{Re} \lambda \geqslant \alpha) .
$$

6.2. COROLLARY. Let $\mu_{-} \in \hat{S}_{K}, c\left(\mu_{-}\right)<1$. Let $\mu_{+} \in M_{0}$ be such that there exists an increasing net $\left(\mu_{L}\right)_{\iota \in I}$ in $S$ such that $\mu_{+}=\sup _{\iota \in I} \mu_{L}$ (in the sense that $\int\left|u^{\sim}\right|^{2} \mathrm{~d} \mu_{+}=\sup _{\iota \in I} \int\left|u^{\sim}\right|^{2} \mathrm{~d} \mu_{\iota}(u \in D) ;$ cf. Theorem 4.5). Then $U_{1,-\mu_{-}+\mu_{+}}(\cdot)$ is holomorphic. (Recall from Theorem 4.1 and Corollary 4.2 that $U_{1,-\mu_{-}+\mu_{+}}(\cdot)$ acts on $L_{1}(Y, m)$ for suitable $Y \subset X$.)

Proof. Using the notation in part (ii) of the proof of Theorem 6.1, we obtain from Theorem 4.5 that, for $\operatorname{Re} \lambda \geqslant \alpha,|\lambda-\alpha|>r$, the resolvent $\left(H_{1,-\mu_{-}+\mu_{+}}+\lambda\right)^{-1}$ is obtained as

$$
\left(H_{1,-\mu_{-}+\mu_{+}}+\lambda\right)^{-1} P=s-\lim _{\iota \in I}\left(H_{1,-\mu_{-}+\mu_{t}}+\lambda\right)^{-1},
$$

where $P$ is the band projection onto $L_{1}(Y, m)$. (This follows from Theorem 4.5 for $\lambda$ with large real part, and carries over to all indicated $\lambda$ by [Ka; Chap. VIII, sec. 1.1].) Therefore, the estimates carry over to the resolvent of $H_{1,-\mu_{-}+\mu_{+}}$.

6.3. COROLLARY. Assume $X$ to be second countable. Then for any $\mu_{+} \in M_{0}$, the semigroup $U_{1,-\mu_{-} \mu_{+}}(\cdot)$ is holomorphic. 
Proof. By [Sto3; Theorem 2.2], the hypothesis of Corollary 6.2 is fulfilled.

\section{Appendix A. A Form Convergence Theorem}

In this appendix we shall prove a simple form convergence theorem which we use repeatedly; this result is implicit in [HS].

We fix a Hilbert space $(\mathcal{H},(\cdot \cdot))$ and a closed symmetric form $\mathfrak{y} \geqslant 1$ on $\mathcal{H}$ with dense domain $\mathcal{K}_{+}$. For the inner product $(\cdot \mid \cdot)_{+}:=\mathfrak{h}[\cdot, \cdot]$, the domain $\mathcal{H}_{+}$is then a Hilbert space. We consider the dual $\mathcal{H}_{-}:=\mathcal{H}_{+}^{*}$, the set of conjugate linear continuous forms dualized via $(\cdot \cdot)$, i.e., with the embedding

$$
\begin{aligned}
J: & \mathcal{H} \rightarrow \mathcal{H}_{-}, \\
& \mapsto(u \mid \cdot) .
\end{aligned}
$$

A.1. THEOREM. In the above situation, let $\mathfrak{b}_{k}$ be closed forms on $\mathcal{H}$ for $k \in$ $\mathbb{N} \cup\{\infty\}$ which satisfy the following conditions:

(i) There exists $c \geqslant 1$ such that $\mathfrak{h} \leqslant \mathfrak{h}_{k} \leqslant c \mathfrak{h}$ for all $k \in \mathbb{N} \cup\{\infty\}$;

(ii) for every $u \in \mathcal{H}_{+}, \mathfrak{h}_{k}[u, \cdot] \rightarrow \mathfrak{h}_{\infty}[u, \cdot](k \rightarrow \infty)$, with respect to the norm topology on $\mathcal{H}_{-}$.

Then $H_{k} \rightarrow H_{\infty}$ in strong resolvent sense, where $H_{k}$ denotes the selfadjoint operator associated with $h_{k}(k \in \mathbb{N} \cup\{\infty\})$.

Proof. We have to show $H_{k}^{-1} \rightarrow H_{\infty}^{-1}(k \rightarrow \infty)$, in the strong operator topology.

For $\tilde{H}_{K}: \mathcal{H}_{+} \rightarrow \mathcal{H}_{-}, \tilde{\mathcal{H}}_{k} u:=h_{k}[u, \cdot]$ it follows that $\left\|\tilde{H}_{k}^{-1}\right\| \leqslant 1$ for all $k \in$ $\mathbb{N} \cup\{\infty\}$. Further $\tilde{H}_{k}^{-1} J=H_{k}^{-1}(k \in \mathbb{N} \cup\{\infty\})$, since for all $u \in \mathcal{H}, v \in \mathcal{H}_{+}$:

$$
\begin{aligned}
\left(H_{k}\left(\tilde{H}_{k}^{-1} J u\right) \mid v\right) & =\mathfrak{h}_{k}\left[\tilde{I}_{k}^{-1} J u, v\right] \\
& =(J u)(v)=(u \mid v) .
\end{aligned}
$$

Consequently,

$$
H_{k}^{-1}-H_{\infty}^{-1}=\tilde{H}_{k}^{-1}\left(\tilde{H}_{\infty}-\tilde{H}_{k}\right) \tilde{H}_{\infty}^{-1} J
$$

Since $\tilde{H}_{k} \rightarrow \tilde{H}_{\infty}$ strongly by condition (ii), the assertion of the theorem follows.

REMARK. Since $\left(\tilde{H}_{k}\right)$ is uniformly bounded, it suffices to check condition (ii) of the preceding theorem for a dense subset of $\mathcal{H}_{+}$.

\section{Appendix B. On the Comparison of Symmetric Semigroups}

In this appendix let $(\Omega, m)$ be a localizable measure space without atoms of infinite measure. Let $H \geqslant 0$ be a selfadjoint operator on $L_{2}(\Omega, m)$ and denote by $\mathfrak{h}$ the 
corresponding form. Then $D:=D(\mathfrak{h})=D\left(H^{1 / 2}\right)$ is a Hilbert space, with the scalar product $(\cdot \mid \cdot)_{\mathfrak{h}}:=\mathrm{h}+(\cdot \cdot \cdot)$. Further assume that $e^{-t H}$ is positivity preserving for all $t \geqslant 0$, or equivalently, by the first Beurling-Deny criterion, that $D$ is a vector lattice (under the order of functions) and $\mathfrak{h}[|u|] \leqslant \mathrm{h}[u]$ for all $u \in D$ (cf. [RS1; Thm. XIII.50], [Da; Thm. 7.16]).

Additionally assume that $\check{D}$ is a closed ideal in $\left(D,\|\cdot\|_{\mathfrak{h}}\right)$; 'ideal': $\check{u} \in \check{D}, v \in$ $D,|v| \leqslant|\check{u}|$ implies $v \in \check{D}$. For Dirichlet forms on second countable spaces $X$, the closed ideals of $D$ have been characterized in [Sto3]; namely

$$
\check{D}=\left\{u \in D ; u^{\sim}=0, \text { q.e. on } X \backslash M\right\}=: D_{0}(M),
$$

for a suitable $M \subset X$.

The following proposition contains the main step of the proof of the subsequent theorem.

B.1. PROPOSITION. In the previous situation let $P: D \rightarrow \check{D}$ be the orthogonal projection. Then

$$
P u \leqslant u \quad\left(u \in D_{+}\right) .
$$

Proof. Let $u \in D, u \geqslant 0, \tilde{u}:=P u$. Then $\|u-\check{u}\|_{\mathfrak{h}} \leqslant\|u-\check{v}\|_{\mathfrak{h}}$ for all $\check{v} \in \dot{D}$,

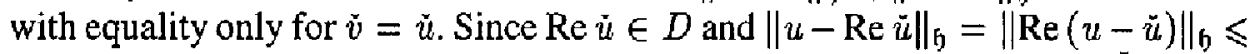
$\|u-\tilde{u}\|_{\mathfrak{h}}$ it follows that $\operatorname{Re} \check{u}=\check{u}$, i.e. $\check{u}$ is real-valued. Further, $u \wedge \check{u} \in \check{D}$ and

$$
\begin{aligned}
\|u-u \wedge \check{u}\|_{\mathfrak{h}}^{2} & =\left\|(u-\check{u})^{+}\right\|_{\mathfrak{h}}^{2}=\mathfrak{h}\left[(u-\check{u})^{+}\right]+\left\|(u-\check{u})^{+}\right\|^{2} \\
& \leqslant \mathfrak{h}[u-\check{u}]+\|u-\check{u}\|^{2}=\|u-\check{u}\|_{\mathfrak{h}}^{2},
\end{aligned}
$$

where the estimate is taken from [RS1; Thm. XIII.50]. We conclude $u \wedge \check{u}=\check{u}$, i.e. $u \geqslant \check{u}$.

Before stating the main result we note that the closure of $\check{D}$ in $L_{2}(\Omega, m)$ is of the form

$$
\left\{f \in L_{2}(\Omega, m) ; f=0 \quad \text { on } \quad \Omega \backslash \check{\Omega}\right\}=L_{2}(\check{\Omega}, m)
$$

where $\check{\Omega}$ is a locally measurable subset of $\Omega$, and the restriction of $m$ to $\check{\Omega}$ is again denoted by $m$. Indeed, it is easily shown that $\check{D}$ is dense in

$$
\left\{f \in L_{2}(\Omega, m) \text {; there exists } u \in \check{D} \text { such that }|f| \leqslant u\right\} \text {. }
$$

Since the latter set is an ideal in $L_{2}(\Omega, m)$ containing $\check{D}$ it follows that its closure, and therefore the closure of $\breve{D}$, is a closed ideal in $L_{2}(\Omega, m)$, which implies that it is of the asserted form (cf. [Sch; Ex.2 on p. 158]). 
Now the restriction $\check{h}$ of $h$ to $\check{D}$ is a closed, positive, densely defined form in $L_{2}(\check{\Omega}, m)$. If we denote by $\breve{H}$ the corresponding selfadjoint operator, then the first Beurling-Deny criterion implies that $e^{-t \check{H}}$ is positivity preserving for all $t \geqslant 0$.

\section{B.2. THEOREM. With the previous notation we have}

$$
e^{-t \hat{H}} f \leqslant e^{-t H} f
$$

for all $f \in L_{2}(\check{\Omega}, m)_{+}, t \geqslant 0$.

Proof. We are going to show

$$
(\check{H}+\alpha)^{-1} f \leqslant(H+\alpha)^{-1} f
$$

for all $f \in L_{2}(\check{\Omega}, m)_{+}, \alpha>0$ which is equivalent to the assertion.

It is sufficient to treat $\alpha=1$ (replacing $\mathfrak{h}$ by $\frac{1}{\alpha} \mathfrak{h}$ ). We observe

$$
(\check{H}+1)^{-1} f=P(H+1)^{-1} f
$$

for all $f \in L_{2}(\check{\Omega}, m)$. In fact, for all $\check{u} \in \check{D}$ we have

$$
\left((H+1)^{-1} f \mid \breve{u}\right)_{b}=(f \mid \check{u})=\left((\check{H}+1)^{-1} f \mid \check{u}\right)_{b} .
$$

Now the desired inequality follows from Proposition B.1.

B.3. COROLLARY. Let $\mathfrak{k} \geqslant 0$ be another closed symmetric form in $L_{2}(\Omega, m)$ (not necessarily densely defined). Assume that $D(\mathfrak{k}) \subset D$ is an ideal in $D$,

$$
\begin{array}{ll}
\mathfrak{k}[|u|] \leqslant \mathfrak{k}[u] & (u \in D(\mathfrak{k})), \\
\mathfrak{b}\{u, v] \leqslant \mathfrak{k}[u, v] & \left(u, v \in D(\mathfrak{k})_{+}\right) .
\end{array}
$$

Then $\overline{D(k)}^{L_{2}}=L_{2}(\dot{\Omega}, m)$ for suitable $\bar{\Omega} \subset \Omega ;$ let $K$ be the selfadjoint operator in $L_{2}(\check{\Omega}, m)$ corresponding to $\mathrm{l}$. Then

$$
0 \leqslant e^{-t K} f \leqslant e^{-t H} f
$$

for all $f \in L_{2}(\check{\Omega}, m)_{+}, t \geqslant 0$.

Proof. Let $\bar{D}:=\overline{D(\mathfrak{l})}^{D}$. Then $\check{D}$ is a closed ideal in $D$. (Ideal: Let $0 \leqslant v \leqslant$ $u, u \in \check{D}, v \in D$. Then there exists $\left(u_{n}\right)$ in $D(\mathfrak{k}), u_{n} \rightarrow u$ in $D$; without restriction $u_{n} \geqslant 0$. Then $v \wedge u_{n} \in D(\mathfrak{k})$, and $v \wedge u_{n} \rightarrow v \wedge u=v$ weakly in $D$.)

Let $\grave{\mathfrak{h}}:=\mathfrak{h} \mid \check{D}, \check{H}$ as previously. Then $0 \leqslant e^{-t \check{H}_{f}} \leqslant e^{-t H} f$ for all $f \in$ $L_{2}(\check{\Omega}, m)_{+}, t \geqslant 0$, by Theorem B.2.

The assumptions imply $e^{-t K^{-}} f \geqslant 0$ for all $f \in L_{2}(\check{\Omega}, m)_{+}, t \geqslant 0$. For $u \in$ $D(K)_{+}(\subset D(\mathfrak{k})), v \in D(\mathfrak{k})_{+}$we have the inequality

$$
\check{\mathfrak{h}}[u, v](\leqslant \mathfrak{k}[u, v]) \leqslant(K u \mid v) .
$$


This inequality extends to all $v \in \dot{D}$, and therefore

$$
(u \mid \check{H} v) \leqslant(K u \mid v)
$$

for all $u \in D(K)_{+}, v \in D(\check{H})_{+}$. This implies $e^{-t K} f \leqslant e^{-t \grave{H}} f\left(f \in L_{2}(\check{\Omega}, m)_{+}\right.$, $t \geqslant 0)$, by [Na; C-II, Proposition 4.8]).

B.4. REMARK. Note that, in Proposition B.1, we did not state that $P$ is positive; this is not true in general. (If, however, $P$ is applied to $(H+1)^{-1} f$ with $f \in$ $L_{2}(\check{\Omega}, m)_{+}$, then the result $(\check{H}+1)^{-1} f$ is positive. $)$

B.5. EXAMPLE. Let $\Omega=\Omega \subset \mathbb{R}^{n}, D:=W_{2}^{1}(\Omega), \mathfrak{b}$ the classical Dirichlet form, $\dot{D}:=W_{2,0}^{1}(\Omega)$. Then in the previous notation, $H$ is the Neumann Laplacian $-\Delta_{N}$ and $\check{H}$ is the Dirichlet Laplacian $-\Delta_{D}$. In this case one obtains $\check{\Omega}=\Omega$, since $W_{2,0}^{1}(\Omega)$ is dense in $L_{2}(\Omega)$. The inequality obtained by applying Theorem B.2 to this situation can also be obtained in a different way; cf. [AB], [Da1; proof of Theorem 2.1.6].

The main results of this appendix, i.e., Theorem B.2 and Corollary B.3, have been extended by Ouhabaz [Ou] to not necessarily symmetric forms.

\section{References}

[ABR] S. Albeverio, J. Brasche and M. Röckner: 'Dirichlet forms and generalized Schrödinger operators', in Schrödinger Operators, Proceedings, Sonderborg, $H$. Holden and A. Jensen (eds.). Springer, Lecture Notes in Physics 345 (1989), 1-42.

[AM] S. Albeverio and Z. Ma: 'Perturbation of Dirichlet forms - Lower semiboundedness, closability, and form cores', J. Funct. Anal. 99 (1991), 332-356.

[AM1] S. Albeverio and Z. Ma: 'Additive functionals, nowhere Radon and Kato class smooth measures associated with Dirichlet forms', Preprint SFB 237, 1989.

[AB] W. Arendt and C. J. K. Batty: Absorption semigroups and Dirichlet boundary conditions. Math Ann. 295 (1993), 427-448.

[Ba] H. Bauer: Maß- und integrationstheorie. W. de Guyter, Berlin, 1990.

[BDM] J. Baxter, G. DalMaso and U. Mosco: Stopping times and $\Gamma$-convergence. Trans. Amer. Math. Soc. 303 (1987), 1-38.

[BM] P. Blanchard and Z. Ma: Semigroup of Schrödinger operators with potentials given by Radon measures. In: Stochastic Processes - Physics and Geometry, S. Albeverio et al. (cds.), Proceedings, Ascona. World Sci., Singapore, 1990.

[BH] N. Bouleau and F. Hirsch: Dirichlet Forms and Analysis on Wiener Space. W. de Gruyler, Berlin, 1991.

[BEKS] J. Brasche, P. Exner, Yu. Kuperin and P. Seba: Schrödinger operators with singular interactions. SFB 237 - Preprint Nr. 132, Bochum, 1991.

[CMS] R. Carmona, W. Ch. Masters and B. Simon: Relativistic Schrödinger operators: Asymptotic behavior of the eigenfunctions. J. Funct. Anal. 91 (1990), 117-142.

[Ca] T. Coulhon: 'Dimension à l'infini d'un semi-groupe analytique', Bull. Sc. Math. $2^{\circ}$ série, 114 (1990), 485-500.

[Da] E. B. Davies: One-Parameter Semigroups. Academic Press, London, 1980. 
[Da1] E. B. Davies: Heat Kernels and Spectral Theory. Cambridge Univ. Press, Cambridge, 1989.

[Dem] M. Demuth: 'On topics in spectral and stochastic analysis for Schrödinger operators', Proceedings Recent Developments in Quantum Mechanics, A. Boutet de Mouvel et al. (eds.), Kluwer, 1991.

[Dev] A. Devinatz: Schrödinger operators with singular potentials. J. Operator Theory 4 (1980), 25-35.

[FIP] T. Figiel, T. Jwaniecz and A. Pelczyński: Computing norms of some operators in $L^{p}$-spaces. Studia Math. 79 (1984), 227-274.

[Fu] M. Fukushima: Dirichlet Forms and Markov Processes. North Holland, Amsterdam, 1980.

[FST] M. Fukushima, K. Sato, and S. Taniguchi: On the closable parts of pre-Dirichlet forms and the fine supports of underlying measures. Osaka J. Math. 28 (1991), 517-535.

[He] L. I. Hedherg; Spectral synthesis and stability in Sobolev spaces, in Euclidean Harmonic Analysis, Proceedings Maryland, J. J. Benedetto (ed.), Lect. Notes in Math. 779 (1980), 73-103.

[HS] I. W. Herbst and A. D. Sloan: Perturbation of translation invariant positivity preserving semigroups on $L^{2}\left(R^{N}\right)$. Trans. Amer. Math. Soc. $236(1978)+325-360$

[HJ] W. Hoh and $N$, Jacob: Some Dirichlet forms generated by pseudo differential operators. Bult. Sci. Math. 116 (1992), 383-398.

[Ja] N. Jacob: Dirichlet forms and pseudo differential operators. Expo. Math. 6 (1988), 313351

[Ka] T. Kato: Perturbation Theory for Linear Operators, 2nd ed., Springer-Verlag, Berlin, 1980.

[Kal] T. Kato: ' $L$-theory of Schrödinger operators with a singular potential', in Aspects of Positivity in Functional Analysis, Nagel, Schlotterbeck, Wolff (eds.), North-Holland, 1986.

[MR] Z. Ma and M. Röckner: An Introduction to the Theory of (Non-Symmetric) Dirichlet-Forms. Springer-Verlag, Berlin, 1992.

[Na] R. Nagel (ed,) One Parameter Semigroups of Positive Operators. Lect. Notes in Math. 1184, Springer-Verlag, Berlin, 1986.

[Ou] E. M. Ouhabaz: 'Propriétés d'ordre et de contractivité des semigroupes avec applications aux opérateurs ellipliques', Thèse de doctorat, Besançon, 1992.

[Pa] A. Pazy: Semigroups of Linear Operators and Applications to Partial Differential Equa. tions. Springer-Verlag, Berlin, 1983.

[RS] M. Reed and B. Simon: Methods of Modern Mathematical Physics. I: Functional Analysis. Revised ed., Academic Press, New York, 1980.

[RS1] M. Reed and B. Simon: Methods of Modern Mathematical Physics. IV: Analysis of Operators. Academic Press, New York, 1978.

[RN] F. Riesz and B. Sz.-Nagy: Vorlesungen uber Funktionalanalysis. VEB Deutscher Verlag d. Wiss., Berlin, 1968.

[Sch] H. H. Schaerfer: Banach Lattices and Positive Operators. Springer-Verlag, Berlin, 1974.

[Si] B. Simon: A canonical demposition for quadratic forms with applications to monotone convergence theorems. J. Funct. Anal. 28 (1978), 377-385.

[Si1] B. Simon: Schrödinger semigroups, Bull. (N.S.) Amer. Math. Soc. 7 (1982), 447-526.

[Sie] E. M. Stcin: Topics in Harmonic Analysis, Related to the Litlewood-Paley Theory. Princeton Univ. Press, Princeton, N.J., 1970.

[Sto] P. Stollmann: Formtechniken bei Schrödingeroperatoren. Diplom thesis Munich, 1985 (unpublished).

[Stoi] P. Stollmann: 'Admissible and regular potentials for Schrödinger forms', J. Operator Theory 18 (1987), 139-151.

[Sto2] P. Stollmann: 'Smooth perturbations of regular Dirichlet forms', Proc. Amer. Math. Soc. $116(1992), 747-752$. 
[Sto3] P. Stollmann: Closed ideals in Dirichlet spaces. Potential Analysis 2 (1993), 263-268.

[Stu] K.-T. Sturm: Measures charging no polar sets and additive functionals of Brownian motion. Forum Math. 4 (1992), 257-297.

[Stu1] K.-T. Sturm: Schrödinger operators with arbitrary nonnegative potentials, in Operator Calculus and Spectral Theory, Proc. Lambrecht 1991, Demuth, Gramsch, Schulze eds., Birkhäuser, 1992, 291-306.

[Va] N. Varopoulos: Hardy-Litlewood theory for semigroups. J. Funct. Anal. 63 (1985), 240260.

[Vo] J. Voigt: On the perturbation theory for strongly continuous semigroups. Math. Ann. 229 (1977), 163-171.

[Vol] J. Voigl: Absorption semigroups, their generators, and Schröỏinger semigroups. J. Funct. Anal. 67 (1986), 167-205.

[Vo2] J. Voigt: Absorption semigroups. J. Operator Theory 20 (1988), 117-131.

[Vo3] J. Voigt: One-parameter semigroups acting simultaneously on different $L_{p}$-spaces. Bull. Soc. Royale. Sc. Liège 61 (1992), 465-470. 